\title{
The Effectiveness of Mindfulness-Based Stress Reduction on the Psychological Functioning of Healthcare Professionals: a Systematic Review
}

\author{
Sarah Angela Kriakous ${ }^{1}$ (D) Katie Ann Elliott ${ }^{2} \cdot$ Carolien Lamers $^{3} \cdot$ Robin Owen $^{4}$ \\ Accepted: 6 September 2020 / Published online: 24 September 2020 \\ (C) Springer Science+Business Media, LLC, part of Springer Nature 2020
}

\begin{abstract}
Objectives Burnout and occupational stress are frequently experienced by healthcare professionals (HCPs). Mindfulness-based stress reduction (MBSR) has been found to improve the psychological health outcomes of HCPs. To date, systematic reviews and meta-analyses have primarily focused upon empirical investigations into the reduction of stress amongst HCPs using MBSR and are limited to empirical studies published before December 2019. This systematic review aimed to update the current evidence base and broaden our understanding of the effectiveness of MBSR on improving the psychological functioning of HCPs.

Methods Three electronic databases (Medline, Psych Info and Web of Science) were searched without time frame restrictions. Quantitative studies included randomised controlled trials, clinical controlled trials, pre-post designs and studies with up to a 12month follow-up period. All studies included in the review employed a MBSR programme, standardised measures of psychological functioning and qualified HCPs as participants.

Results Using PRISMA guidelines thirty studies were included in the review. The reviewed literature suggested that MBSR was effective in reducing HCPs experiences of anxiety, depression and stress. MBSR was also found to be effective in increasing HCP levels of mindfulness and self-compassion. However, MBSR did not appear as effective in reducing burnout or improving resilience amongst HCPs. Abbreviated MBSR programmes were found to be as effective as the traditional 8-week MBSR programmes.

Conclusions MBSR is an effective intervention which can help improve the psychological functioning of HCPs. Recommendations include improving the overall quality of the studies by employing more robust controlled designs with randomisation, increased sample sizes with heterogeneous samples, and making active comparisons between interventions used.
\end{abstract}

Keywords Mindfulness $\cdot$ Mindfulness-based stress reduction $\cdot$ Stress $\cdot$ Burnout $\cdot$ Resilience $\cdot$ Review

HCPs are exposed to emotionally challenging and stressful situations in the workplace, particularly in the current climate,

Sarah Angela Kriakous

drsarahkriakous@gmail.com

1 Elysium Healthcare Limited, Ty Grosvenor, 16 Grosvenor Road, LL11 1BU Wrexham, UK

2 Forensic Clinical Psychology Department, North Wales Forensic Psychiatric Service, Ty Llywelyn Medium Secure Unit, Ysbyty Bryn y Neuadd, Llanfairfechan, LL33 0HH Conwy, UK

3 North Wales Clinical Psychology Programme, Bangor University, LL57 2DG Bangor, UK

4 Early Interventions in Psychosis, Hergest Unit, Ysbyty Gwynedd, Gwynedd, LL57 2PW Bangor, UK with an increased demand for clinical productivity and other work-related pressures (Schinler et al. 2006). Occupational stress and burnout are common amongst different HCPs, including nurses, physicians and psychologists (Rupert and Morgan 2005; Shanafelt et al. 2003; Vahey et al. 2004). Stress can be defined as a relationship between an individual and their environment, which the individual appraises as potentially threatening to their well-being (Lazarus and Folkman 1984).

In relation to burnout, there does not appear to be a universally agreed definition (Farber 1983). However, burnout can be viewed as a process rather than a fixed state (Schulz et al. 1995) and defined as emotional, mental and physical exhaustion caused by excessive and prolonged stress. In relation to employment, Maslach et al. (1996) developed a model of burnout which they described as a work-related 
syndrome with three components: (1) emotional exhaustion, (2) depersonalisation and (3) diminished feelings of personal accomplishment. Emotional exhaustion refers to employees having a reduced ability to experience emotions relating to their work. Depersonalisation is a process which involves employees distancing themselves from their patients by disregarding the characteristics that make them unique. Personal accomplishment relates to employees' feelings of achievement from working with patients (Maslach et al. 1996, 2001).

Occupational stress and burnout amongst HCPs have been associated with physical and mental health problems, including anxiety, depression, diabetes, fatigue, heart disease, hypertension, insomnia and obesity (Byrant et al. 2000; Miller et al. 1988; Spickard et al. 2002; Weinberg and Creed 2000). Burnout amongst HCPs has also been associated with reduced job satisfaction (Dougherty et al. 2009) and negative patient outcomes, including reduced patient satisfaction and increased work errors (Fahrenkopf et al. 2008; Vahey et al. 2004; Williams et al. 2007).

There is a growing body of research evidence which has suggested that mindfulness-based interventions (MBIs) can help decrease stress and burnout experienced amongst HCPs, increase job satisfaction levels and improve patient outcomes (Escuriex and Labbe 2011; Irving et al. 2009; Shanafelt et al. 2009). Although there are varying definitions of mindfulness, it is commonly and operationally defined as the quality of awareness that occurs through intentionally focusing on present moment experiences in an accepting and non-judgemental manner (Kabat-Zinn 1994).

Mindfulness has become an increasingly popular psychological intervention worldwide; it has an extensive and well-established evidence base which has investigated the efficacy of MBIs to improve the psychological functioning and well-being in both clinical and non-clinical populations (Gu et al. 2015). There are numerous MBIs that are currently being employed with clinical and non-clinical populations; however, mindfulness-based stress reduction (MBSR; Kabat-Zinn 1982) is one of the most extensively used and evaluated MBIs. MBSR was originally designed for patients with chronic medical conditions to help reduce stress and improve their quality of life via focused attention, meditation, cognitive restructuring and adaptive learning techniques (Kabat-Zinn 2013). The standard MBSR programme is an 8-week group intervention, where participants meet once weekly for 2 to $3 \mathrm{~h}$ and undertake a 6-h silent retreat. Participants are encouraged to complete $45 \mathrm{~min}$ of daily mindfulness practice to aid skill generalisation (Virgili 2013). Mindfulness practices are taught via formal practices (e.g. body scan, sitting meditation, mindful walking and hatha yoga) and informal practices whereby individuals are instructed to mindfully engage in typically mindless tasks (e.g. brushing teeth and washing dishes).
There are numerous theoretical models to explain the potential mechanisms of mindfulness and MBIs. A similarity of many of the models is that they postulate that mindfulness causes a positive shift in perspective and an ability to objectively view one's life experiences, this is often referred to as "decentering" (Baer 2003; Brown et al. 2007; Shapiro et al. 2006). Shapiro et al. (2006) highlighted that mindfulness involves three interwoven mechanisms that form part of a single cyclical process: (1) intention (on purpose), (2) attention (paying attention) and (3) attitude (with openness and non-judgement). Furthermore, these three mechanisms of mindfulness are conceptualised as a single cyclical process and lead to the aforementioned shift in perspective, which Shapiro et al. (2006) referred to as 'reperceiving'. Reperceiving facilitates an individual's ability to observe their thoughts and feelings with greater clarity. Reperceiving is classified as a metamechanism that then leads to an additional four mechanisms: (1) self-regulation; (2) values clarification; (3) cognitive, emotional and behavioural flexibility; and (4) exposure to strong emotions with objectivity. These additional mechanisms result in changes which then produce positive outcomes (e.g. adaptive coping and reduction in stress).

Kabat-Zinn (2013) suggested that individuals can react to stress in a habitually unhealthy way. These feelings and behaviours are referred to as a 'habitual or automatic stress reaction' (Kabat-Zinn 2013). Kabat-Zinn (2013) further highlighted that mindfulness can help individuals employ a 'mindfulness-mediated stress response'. This process enables individuals to engage in the process of reperceiving, this then reduces the power of the stress reaction, and consequently, individuals can employ more adaptive, effective coping strategies, which in turn reduces stress. With regard to HCPs, the practice of mindfulness encourages a person to 'check-in' with their own inner experiences and to better understand their personal strengths, difficulties and best contributions (Pipe et al. 2016). Bishop et al. (2004) proposed a two-component model of mindfulness: self-regulation of attention and acceptance of one's own experiences (non-reactive awareness). Arguably, non-reactive awareness may help HCPs facilitate the process of 'decentering' and 'reperceiving' in the workplace. With the development of such skills, HCPs could better manage stress and thus reduce stress and burnout levels.

Over the past decade, research attention has increasingly focused upon the effectiveness and utility of MBSR programmes and several systematic reviews and metaanalyses have been completed. For example, two reviews conducted with a population of healthy adults concluded that, despite available studies being flawed with methodological limitations, MBSR was efficacious in reducing both anxiety and stress and increasing self-compassion (Chiesa and Serretti 2009; Sharma and Rush 2014). Similarly, a meta-analysis concluded that brief versions (e.g. 4-6 weeks) of MBSR were equally as effective in reducing psychological distress in 
working adults compared with the MBSR originally designed for clinical populations (Virgili 2013).

Specific reviews have investigated the effectiveness of MBIs on the psychological functioning of HCPs and have concluded that MBIs were effective in increasing mindfulness and self-compassion and reducing anxiety, burnout, stress and rumination (Escuriex and Labbe 2011; Irving et al. 2009; Morgan et al. 2014; Smith 2014). However, the MBIs included in these reviews were not all based on MBSR (Escuriex and Labbe 2011). They were a mixture of qualitative and quantitative studies (Escuriex and Labbe 2011; Irving et al. 2009; Morgan et al. 2014; Smith 2014) and the samples included specific groups of HCPs (e.g. nurses). Furthermore, the variability of the studies included suggested that there was a need for future reviews to focus specifically on the effectiveness of MBSR on the psychological outcomes of different groups of HCPs. Consistent with this, Irving et al. (2009) undertook a review of ten empirical studies examining the potential benefits of MBSR in improving well-being and coping with stress amongst HCPs. From the ten quantitative studies reviewed, Irving et al. (2009) found that MBSR consistently reduced anxiety, emotional exhaustion and stress, whilst also increasing positive affect. However, the review only included published studies until 2007, and many of the studies were noted to have methodological shortcomings, such as small sample sizes and only one study included an additional treatment comparison.

A systematic review and meta-analysis by Burton et al. (2017) included of seven studies which focused on the benefits of MBIs reducing stress in HCPs. Findings from this review suggested that MBIs had the potential to significantly reduce stress experienced amongst HCPs. Although the authors argued that the quality of some studies proved high with regard to their clarity of aims, data collection and analysis, they also found that some studies continued to present with methodological shortcomings, including small sample sizes and limited use of theoretical frameworks. It was concluded that future research needed to investigate the sustaining effects of mindfulness training on stress experienced amongst HCPs. The review included studies published before August 2015.

More recently, Lomas et al. (2019) completed a systematic review and meta-analysis of forty-one studies which had investigated the effectiveness of MBIs on HCPs wellbeing and included both negative (e.g. anxiety, depression and stress) and positive (e.g. life satisfaction and emotional intelligence) outcome measures. The review concluded that MBIs were associated with positive outcomes in relation to most measures collected and appeared to improve the well-being of HCPs. However, the quality of the studies proved inconsistent and future high-quality randomised control trials (RCTs) were recommended. The review included studies published before January 2016.
Furthermore, Ruiz-Fernandez et al. (2019) also completed a systematic review and meta-analysis of six controlled studies which investigated the effectiveness of types of MBIs in reducing stress and improving self-compassion and mindfulness amongst HCPs. The review highlighted that MBSR was the most commonly used type of MBI. Consistently, MBIs were found to reduce stress experienced amongst HCPs and increased mindfulness. However, the review is noted to have only included controlled studies published before March 2018.

Finally, Spinelli et al. (2019) completed a meta-analysis of thirty-eight RCTs which focused on the effect of mindfulness on both qualified and trainee HCPs. The review highlighted that mindfulness helped significantly reduce anxiety, depression, psychological distress and stress levels. Although the effect was smaller, significantly reduced burnout levels were also observed. The review highlighted the importance of assessing participant needs prior to selecting MBI type and that future studies should focus on measuring changes in mindfulness and include active controls. However, only RCTs published before August 2018 were included in this review.

More recent systematic reviews and meta-analyses have not quality assessed a number of existing studies in their reviews, due to their focus being on controlled studies and depending on the similarity of outcome measures so that meta-analytic procedures can be completed. Since the completion of the aforementioned reviews additional studies have been published in this area. Therefore, the systematic review aimed to examine controlled and uncontrolled quantitative studies that investigated the effectiveness of MBSR on the psychological functioning of HCPs more broadly. In addition, the systematic review also examined additional factors which may have influenced MBSR treatment outcomes, namely the nature of self-selected samples, level of mindfulness home practice, participant incentives and treatment dosage and duration. Robust theoretical frameworks and how they apply to the role of mindfulness to HCPs were also considered.

\section{Method}

\section{Search Strategy}

The systematic review provided an update of Irving et al. (2009) original review. The search was completed in January 2020. Three electronic databases (Psych Info, Medline and Web of Science) were searched without any timeframe restrictions and studies up to December 2019 were included in the search. For the purpose of the review, studies published before 2008 were not included, as no other studies were identified from the 
search pre-2008 that had not already been included in other reviews.

Search terms related to the intervention (mindfulness) and the participant group (healthcare professionals/health care professionals/healthcare workers/health care worker) were used for all three databases. The filter peer-reviewed studies were used where possible. Initially, duplicate studies from the three different databases searches were identified and removed accordingly. Titles and abstracts of eligible studies were identified and reviewed. Studies that did not meet the minimum inclusion criteria were eliminated. Remaining relevant citations were obtained in full text and assessed in relation to the inclusion/exclusion criteria, thus identifying a final list of studies for inclusion in the systematic review.

Other resources included in the search strategy were the Cochrane Library, the Mindfulness Journal and the American Mindfulness Research Association's Mindfulness Research Monthly Newsletter. Figure 1 illustrates the PRISMA diagram (Moher et al. 2009) detailing the different stages of the search strategy for the review.

\section{Inclusion and Exclusion Search Criteria}

\section{Types of Studies}

Studies included in the review had a quantitative methodology or mixed methods (quantitative and qualitative) methodology. Studies were excluded if they solely had a qualitative methodology. Studies had a controlled or uncontrolled pre-post design and included the use of control groups, waiting-list controls or active treatment comparisons. Studies included were published in the English language and from peer reviewed publications.

\section{Types of Participants}

Studies included had samples of qualified HCPs working within an academic, clinical and voluntary capacity. Professional disciplines included counsellors, nurses, different specialisms of physicians, mental health workers, occupational therapists, psychologists, psychiatrists, social workers and support staff working within a healthcare setting. Students and in-training HCPs were excluded due to this population
Fig. 1 PRISMA flow diagram of the different phases of the systemic review, reproduced from Moher et al. (2009) (p.3)

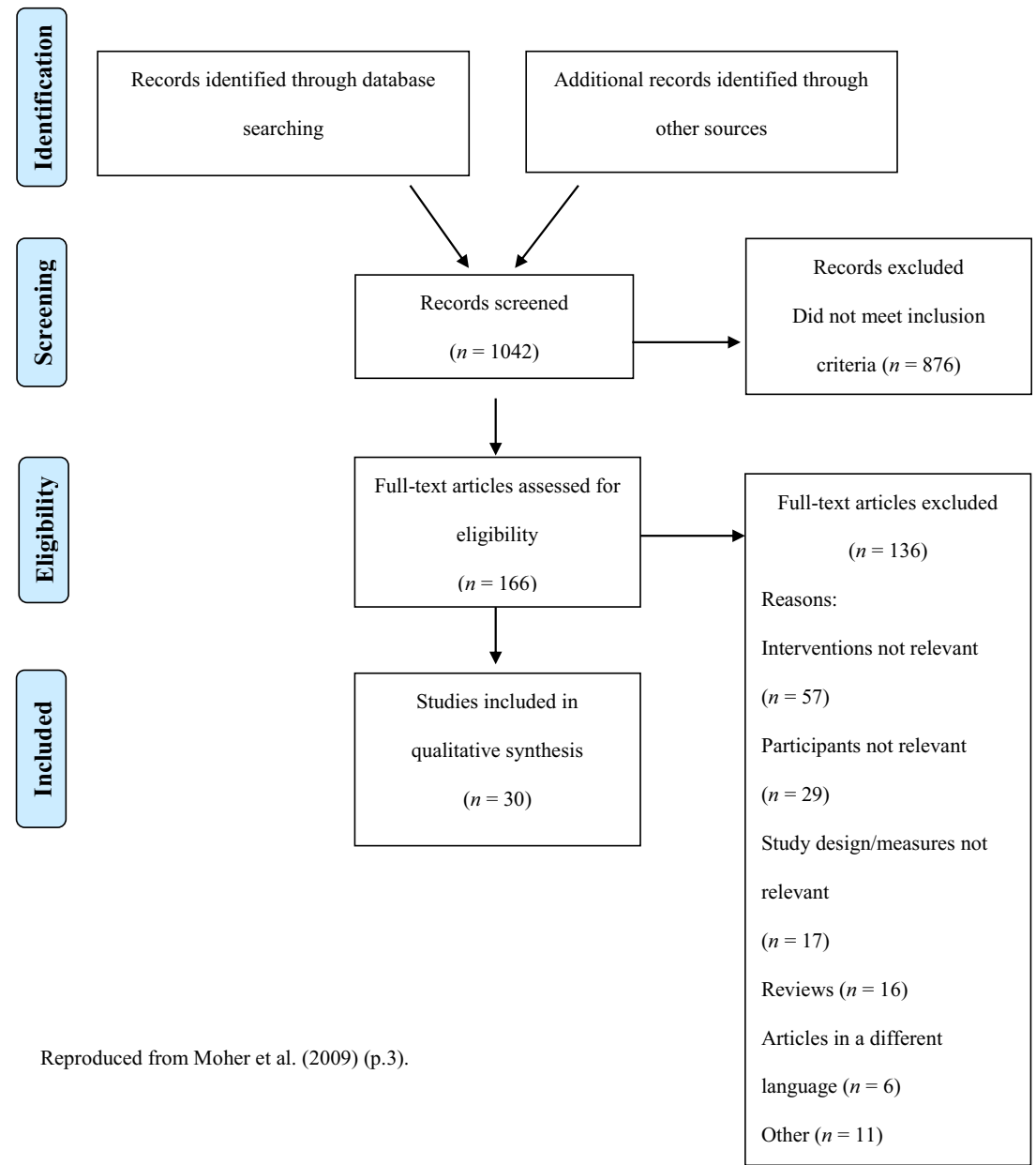


arguably having differing stressors to qualified HCPs (e.g., exam conditions, deadlines for assignments and managing academic and clinical demands). The aim of the review was to build on previous reviews by broadly including HCPs; therefore, it was deemed more helpful to keep the stressors relatively similar. Studies were excluded if the sample were carers for friends and/or family members.

\section{Types of Interventions}

Studies included used MBSR and any modified programmes derived directly from MBSR (e.g. different programme duration). Studies were excluded if the content of the MBSR programme included additional therapeutic concepts, such as acceptance and commitment therapy (ACT; Hayes and Wilson 1994), dialectical behaviour therapy (DBT, Linehan, 1993) and mindfulness-based cognitive therapy (MBCT; Segal et al. 2002). Further, MBSR programmes that included additional psycho-educational components related to occupational functioning were excluded (e.g. burnout and leadership). Studies were also excluded if an audio CD of guided mindfulness practice was used solely as the mode of intervention. Only including MBSR studies strengthened the review because it allowed for a more direct comparison with MBSR abbreviated versions and it also allowed for more homogeneity, thus extrapolating findings to a likely effective component of the intervention. No restrictions were applied for the duration of MBSR interventions or the use of adapted MBSR interventions.

\section{Types of Outcome Measures}

Outcome measures were administered pre-treatment, posttreatment and where relevant for some studies follow-up. At least one standardised measure for an area of psychological functioning was used. Psychological functioning was defined as an individual's ability to achieve their goals internal and external to themselves, and included the individual's mood, emotions, mental health and behaviour (Preedy and Watson 2010). Thus, outcome measures included measures of anxiety, depression, stress, resilience, burnout, self-compassion, mental well-being, psychological distress and mindfulness. Outcome measures and subscales were excluded if they measured empathy or compassion towards others, physical health, rumination or spirituality.

\section{Quality Assessment Tool}

In order to assess the quality of the identified papers, the Quality Assessment Tool for Quantitative Studies (QATQS) (National Collaborating Centre for Methods and Tools 2008) was used. The QATQS was developed by the Effective Public Health Practice Project (EPHPP 1998) and has been found to have good content validity and test-retest reliability (Thomas et al. 2004).

The QATQS measures the methodological rigour of studies with regard to six components: (1) selection bias, (2) study design, (3) confounders, (4) blinding, (5) data collection methods and (6) withdrawal and drop outs. Two final components, intervention integrity and analysis, are also incorporated into the QATQS but a score is not provided for these sections. Each area is assessed on a quality score of 1 to 3 ( 1 = strong; 2 =moderate; 3 =weak). Scores for each area are collated and a global score assigned to each study. If there are no weak ratings and four strong ratings the study scored 'strong'; one weak rating and less than four strong ratings the study scored 'moderate'; and two or more weak ratings then the study scored 'weak'.

QATQS scoring was conducted primarily by the first author, following the guidelines outlined in the QATQS protocol. A dictionary specific to the QATQS is used to maintain standardised results. Inter-rater reliability of the quality assessment of all the studies was examined between the first and third authors. Deviations in how aspects of QATQS were interpreted were noted; however, following further discussion, no deviations in interpretation were noted.

\section{Results}

In total, thirty studies were identified and included in the review using the search method and inclusion and exclusion criteria detailed above.

\section{Study Quality}

The QATQS was employed to review the quality and robustness of the thirty research studies included in the review. The 'global quality rating' results indicated that six studies were assessed as 'moderate to strong', five studies as 'moderate' and nineteen studies as 'weak'. A summary of the thirty studies is presented in Table 1. The studies have been ordered with the most rigorous studies first based on the overall global quality rating, along with the RCTs and studies with control groups also ordered first.

\section{Study Characteristics}

Four studies employed an RCT design, nine studies employed a clinical controlled trial (CCT), one study employed a quasiexperimental design and the sixteen remaining studies employed a pre-post design with no control or comparison treatment groups. Three studies utilised a mixed methods design, including quantitative and qualitative data. Six studies had a control group, six studies used a waitlist control group, one study had a control group which offered an advanced 


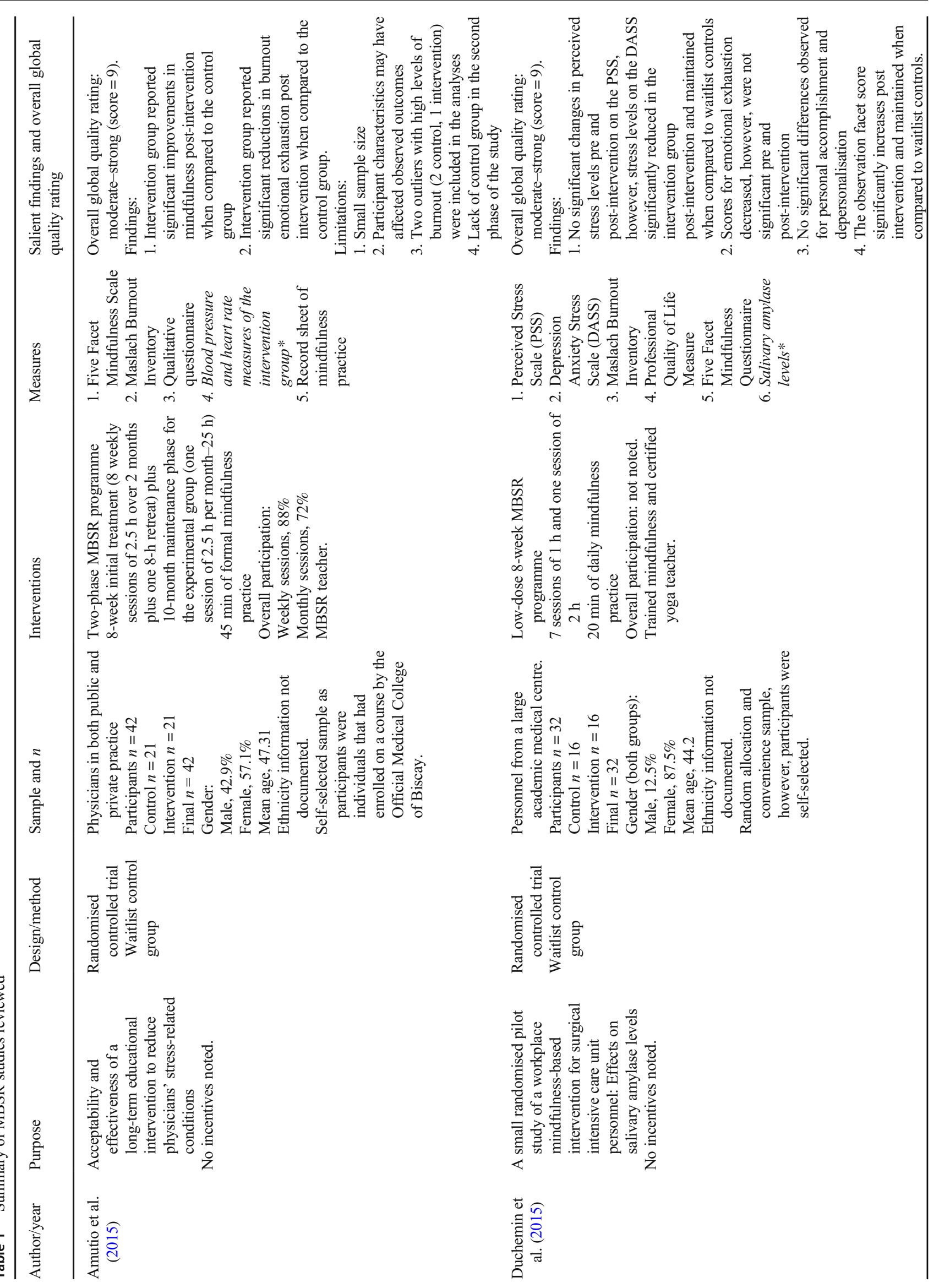




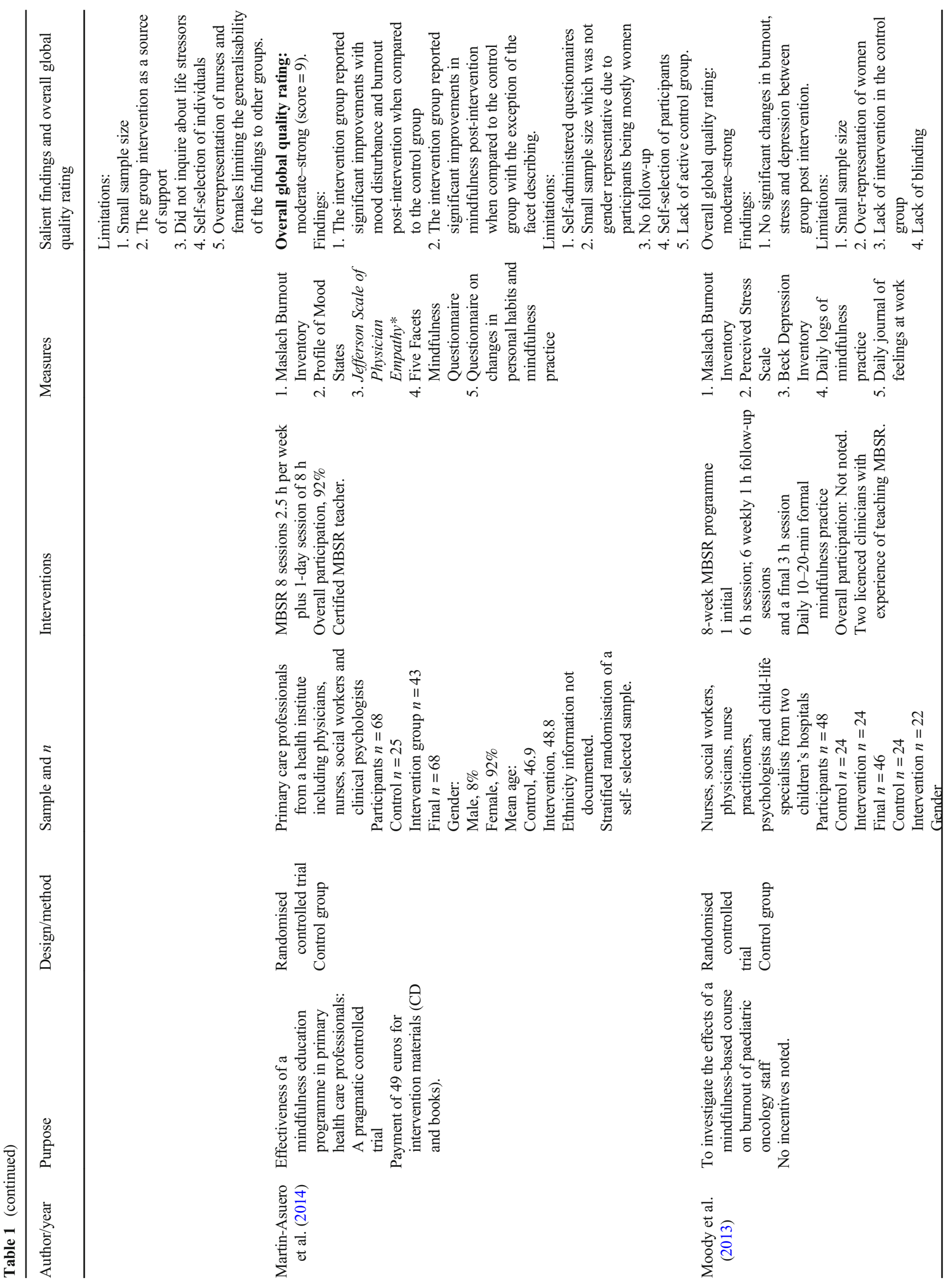




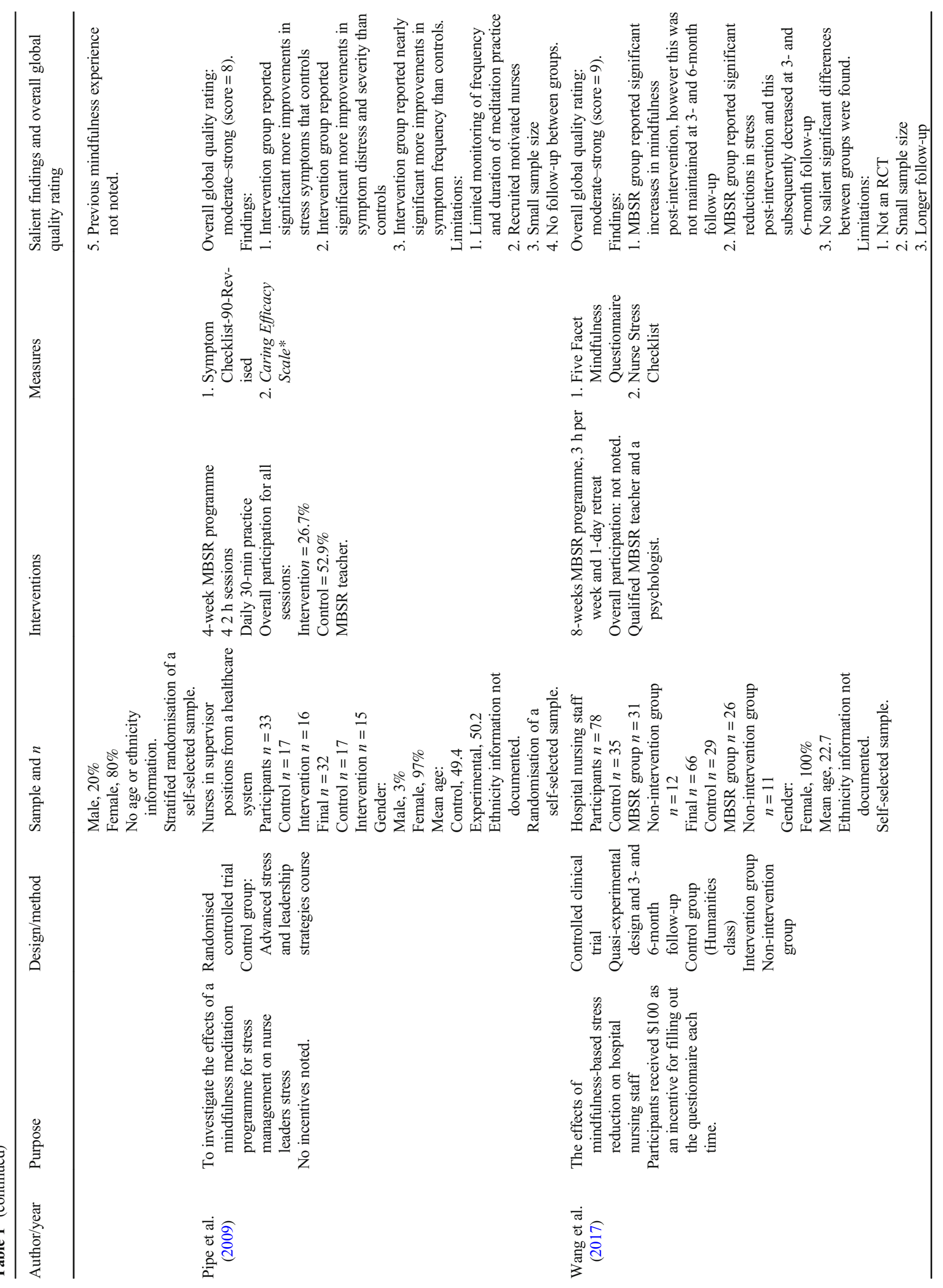


专。몽

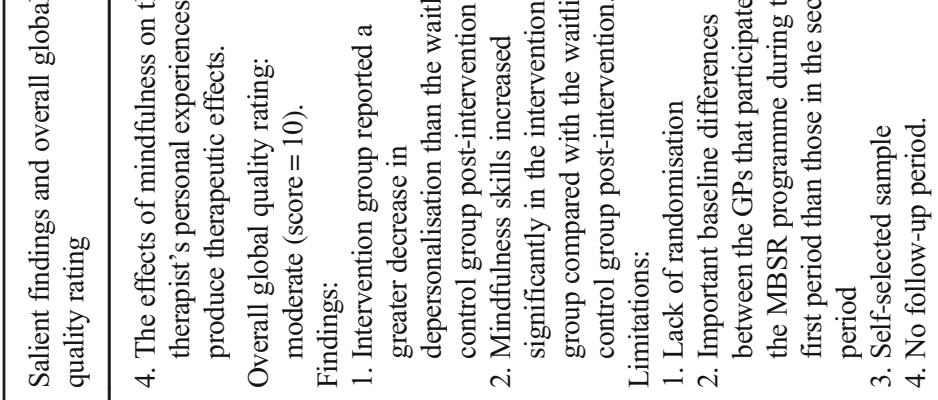

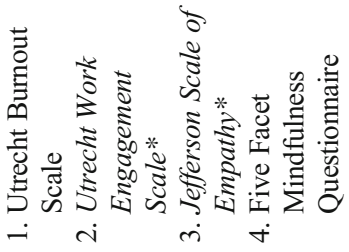

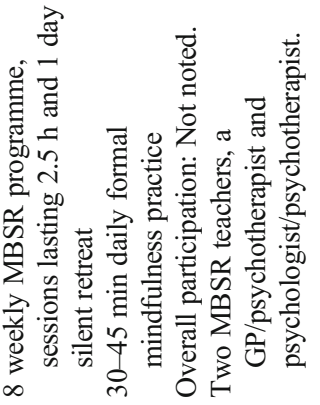

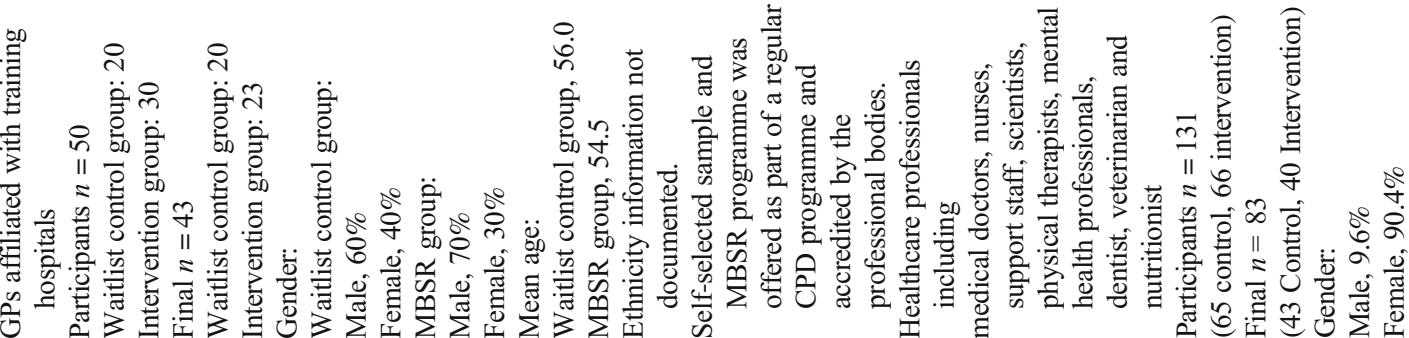

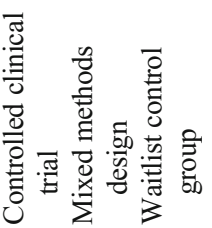

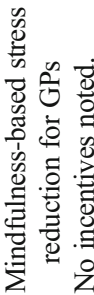

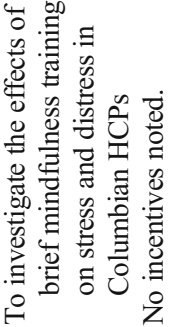

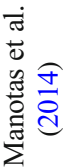

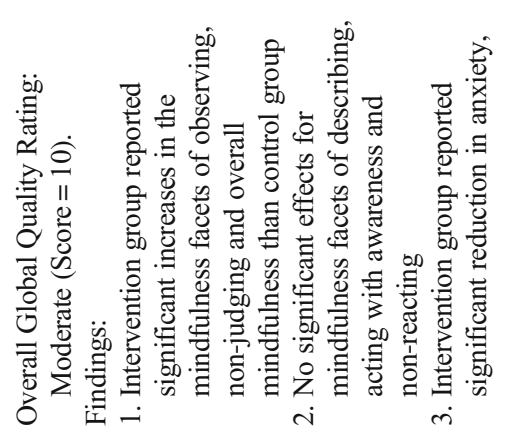

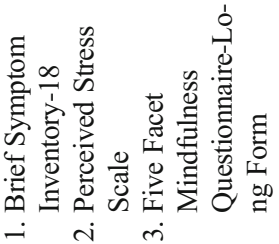

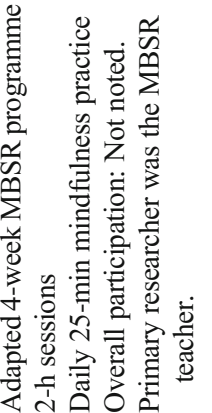

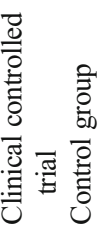

: 


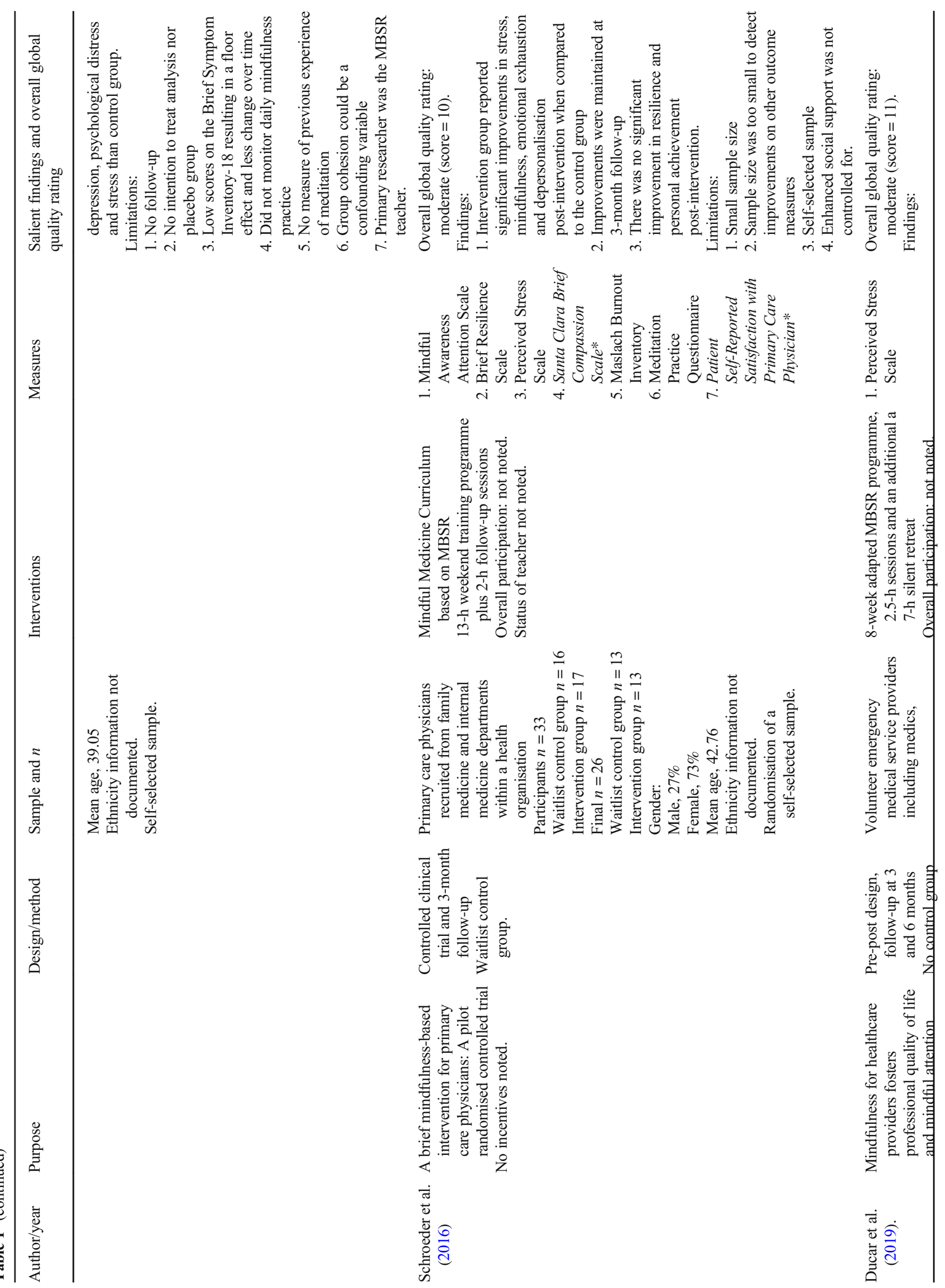




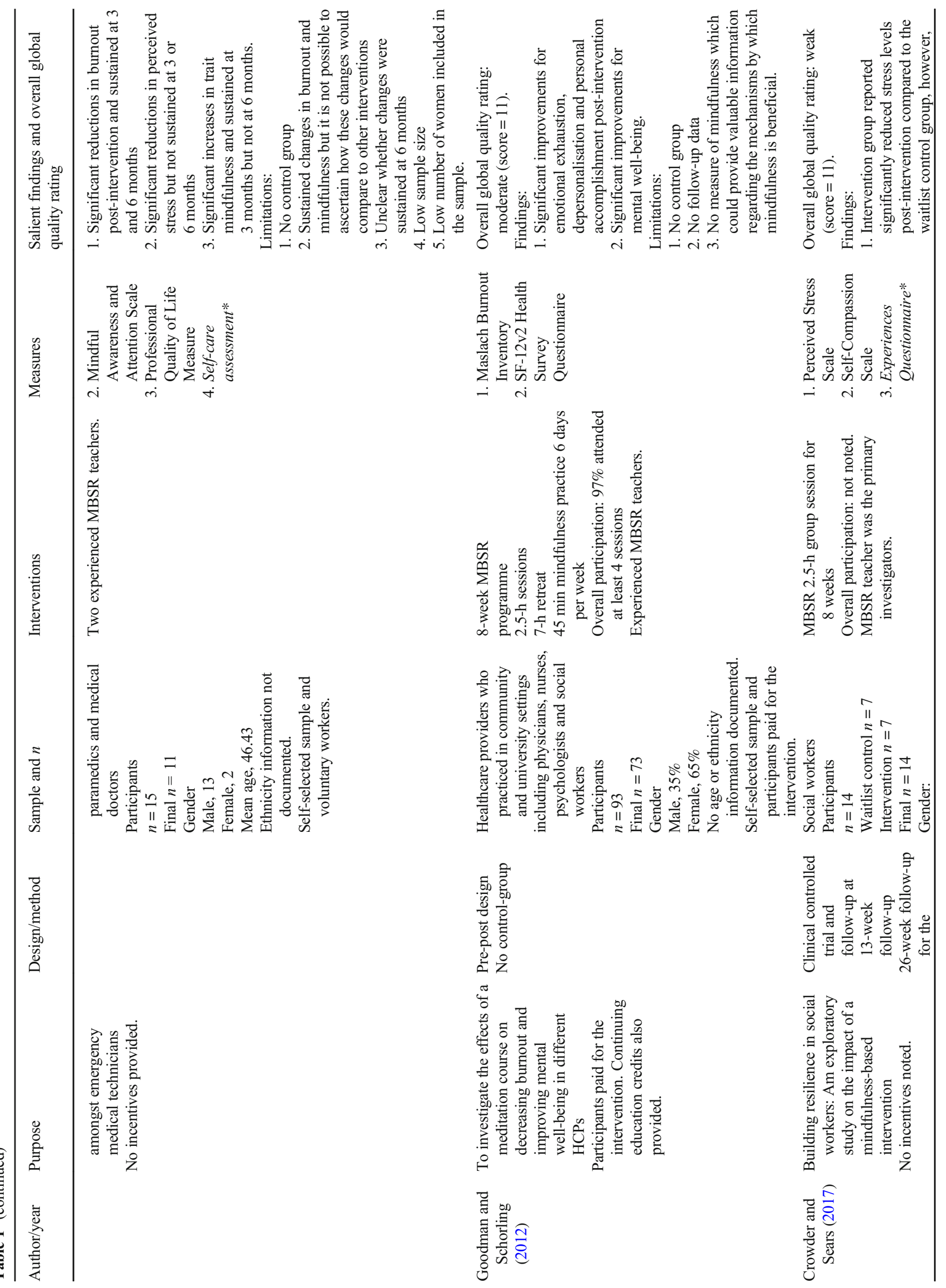




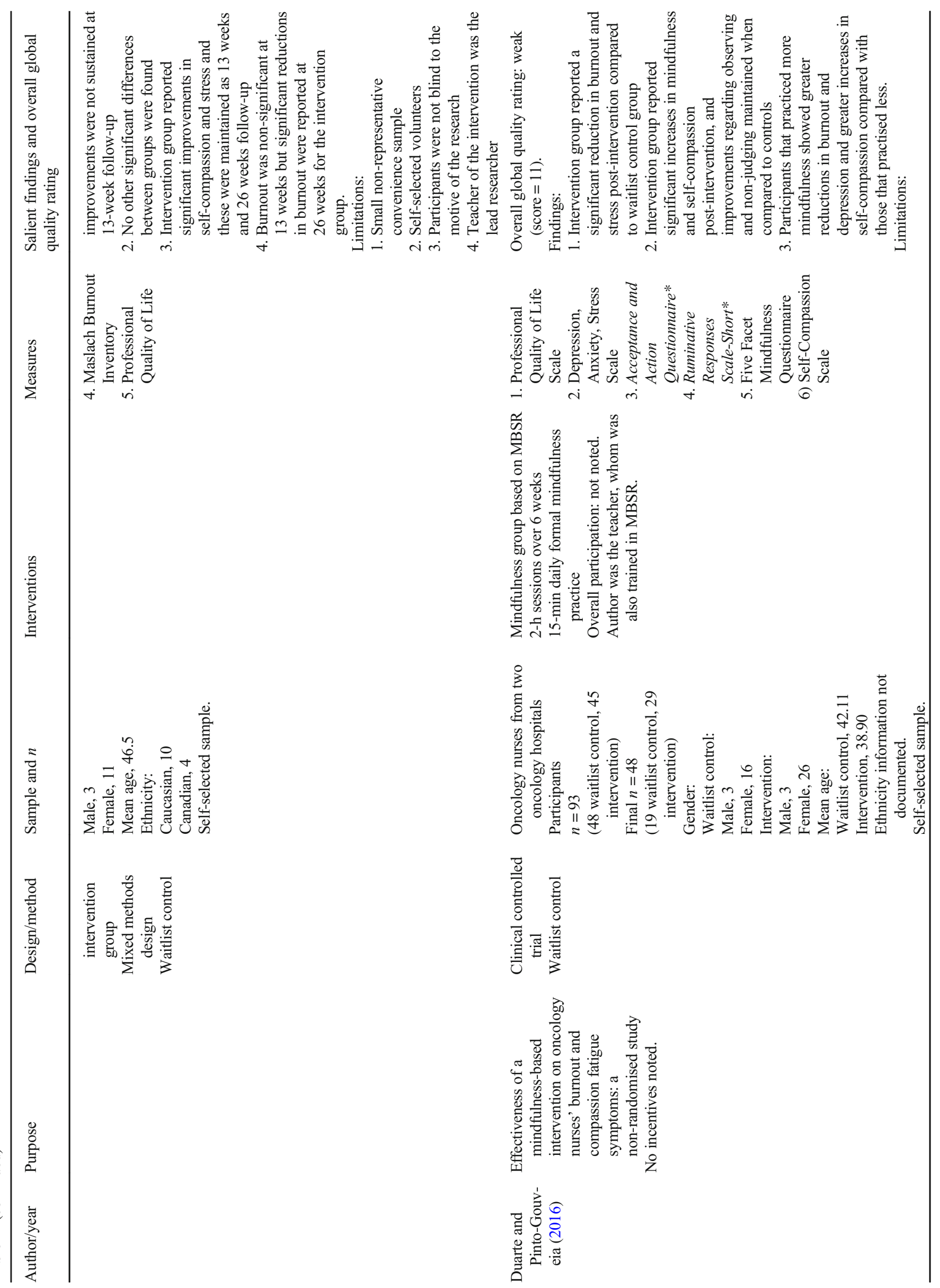




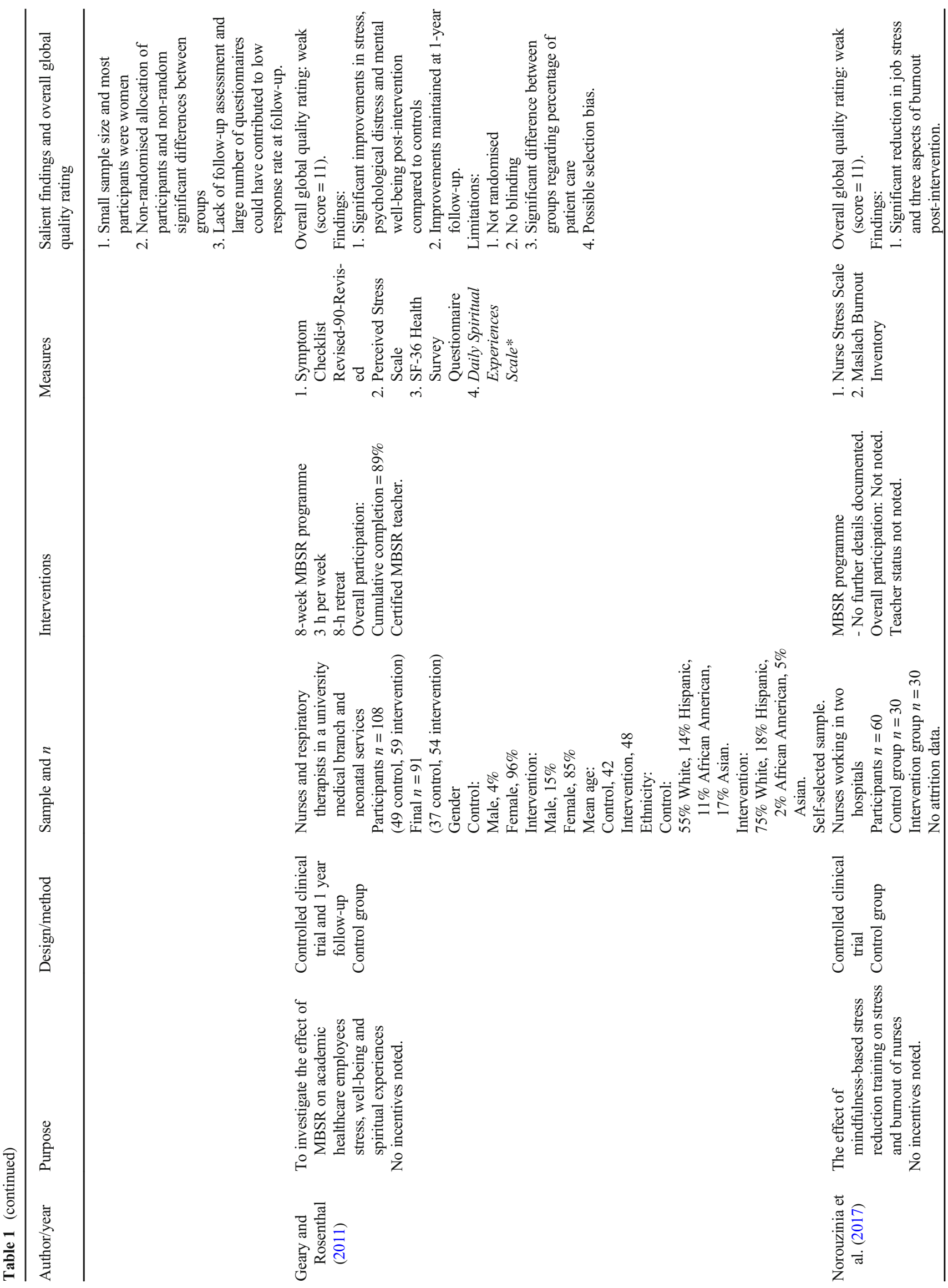




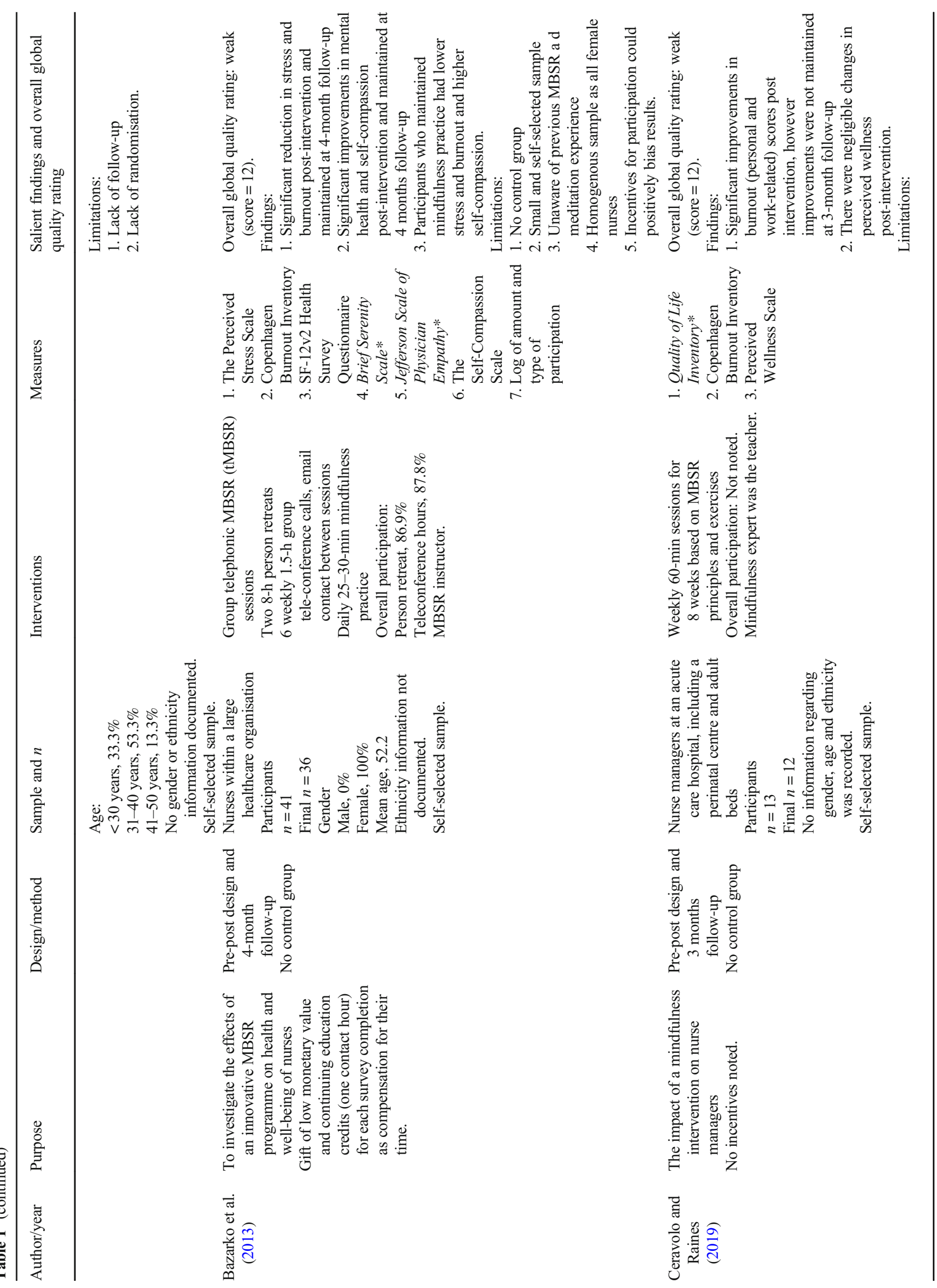




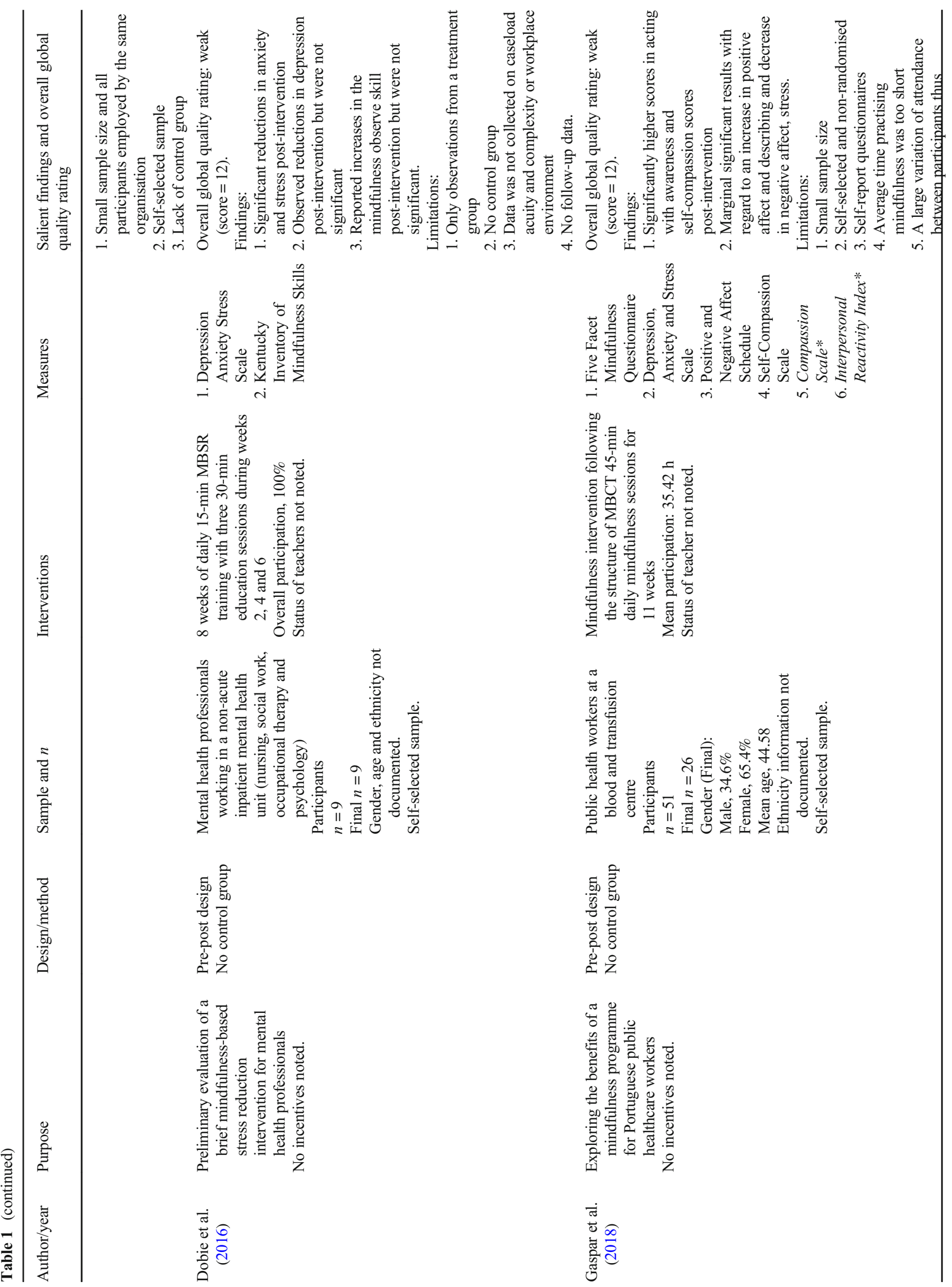




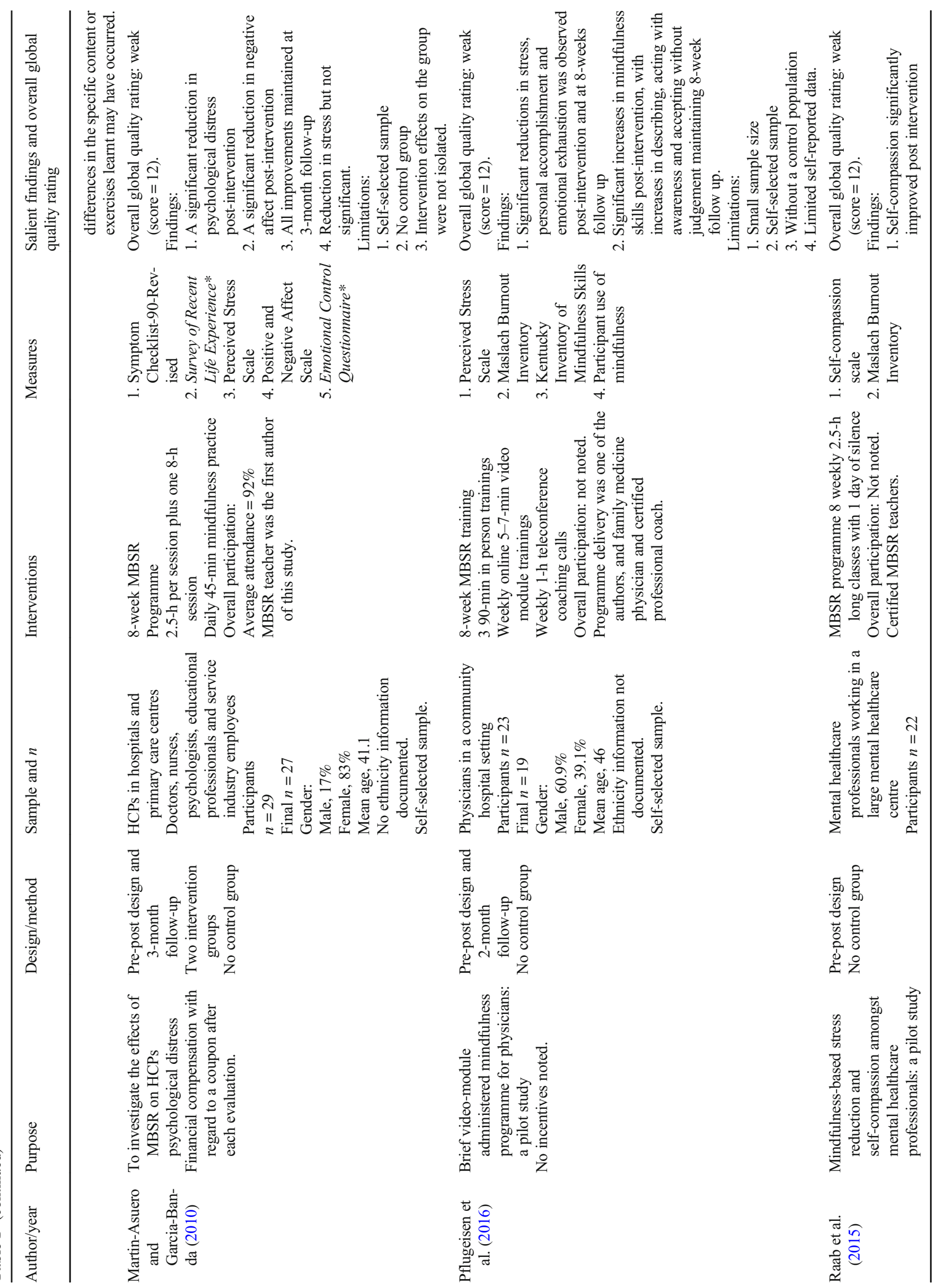




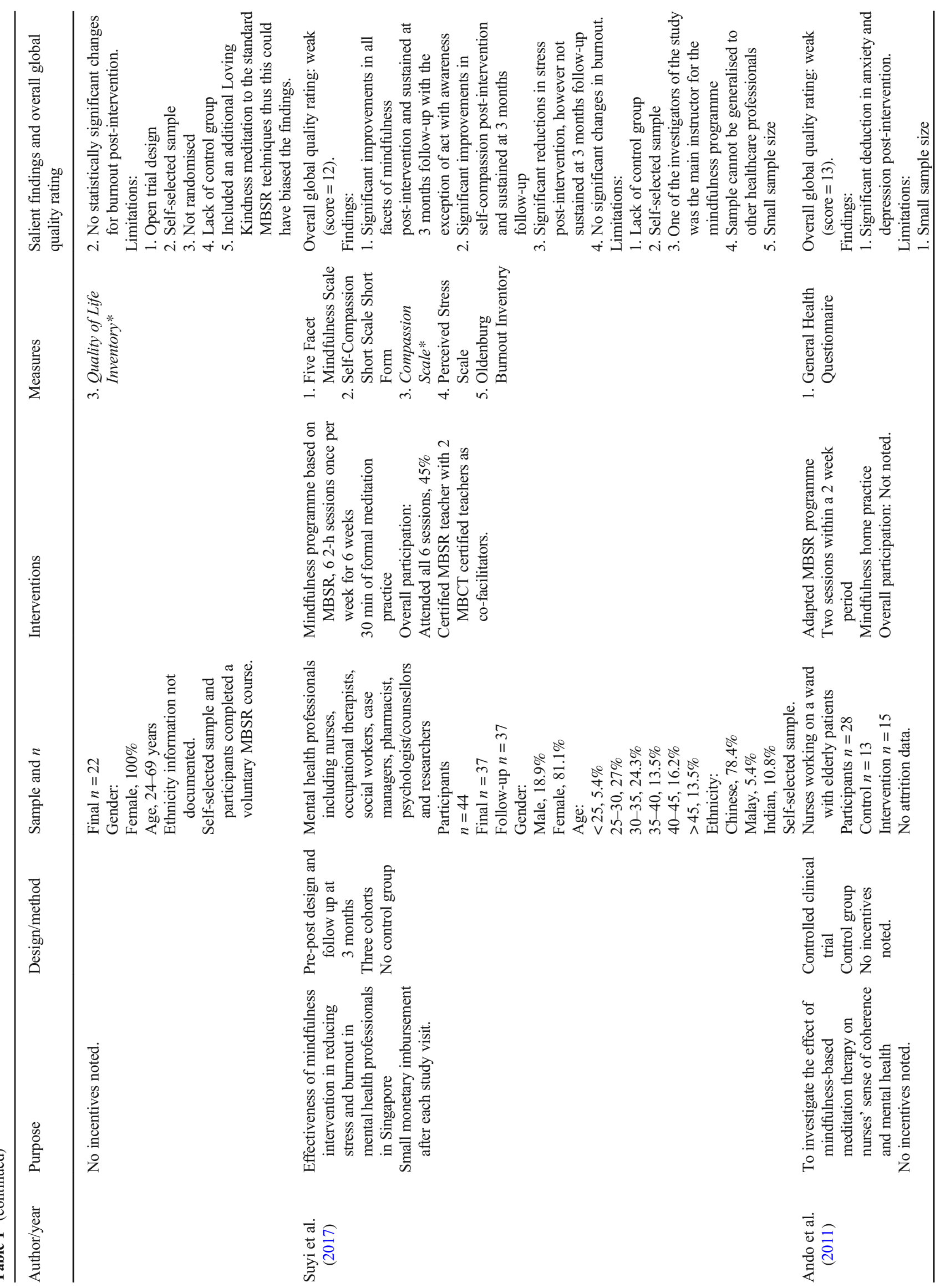




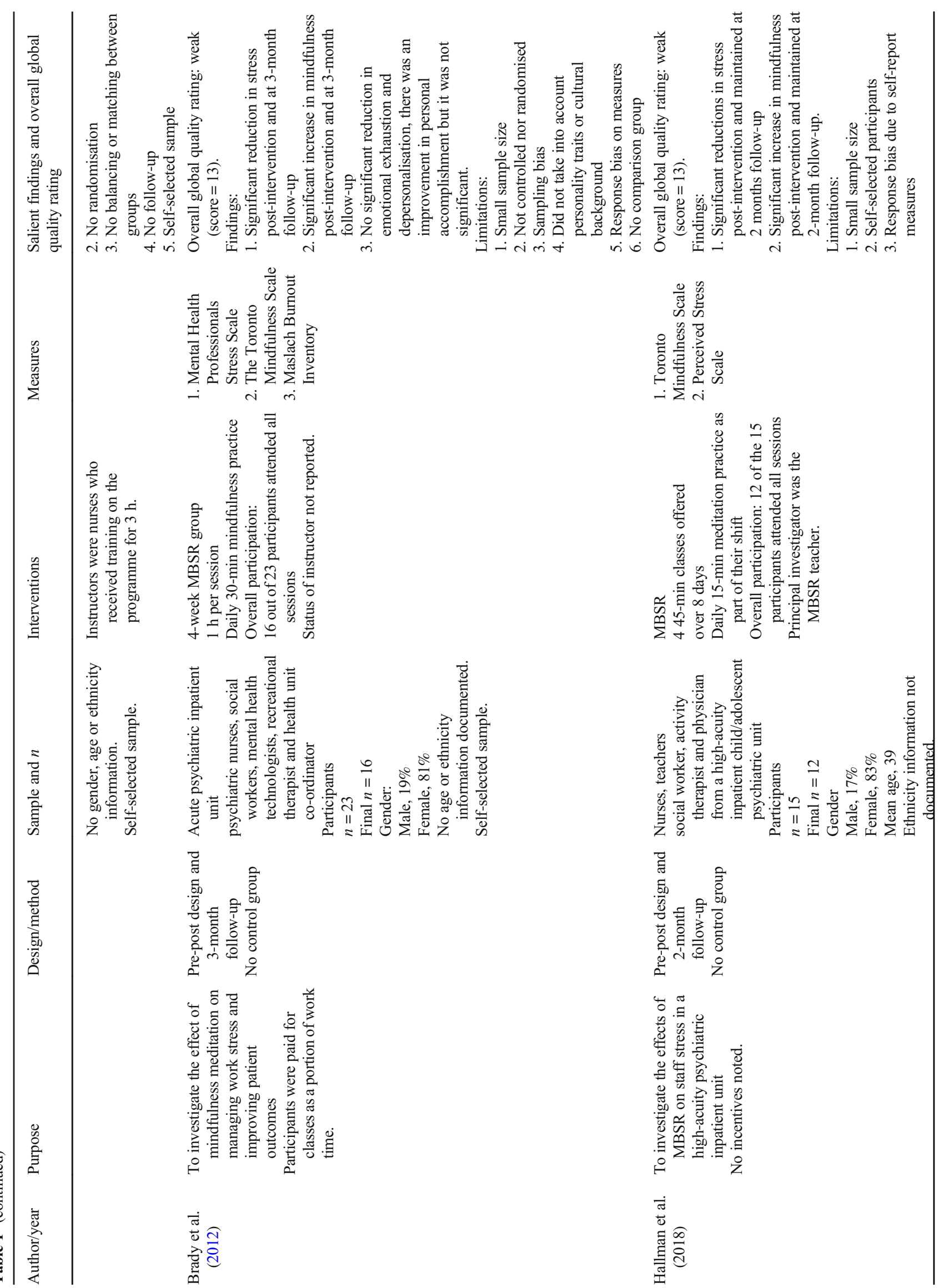




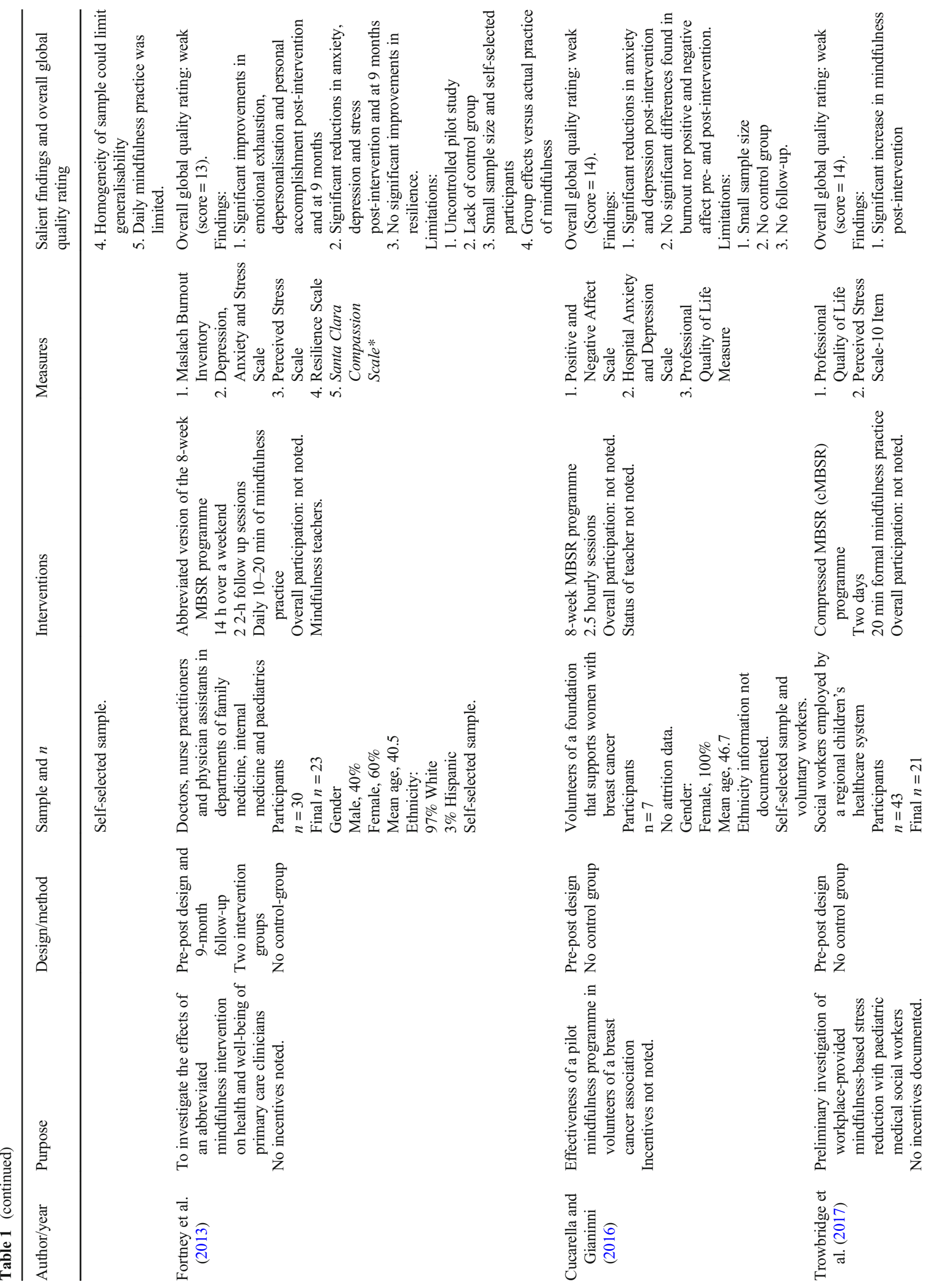




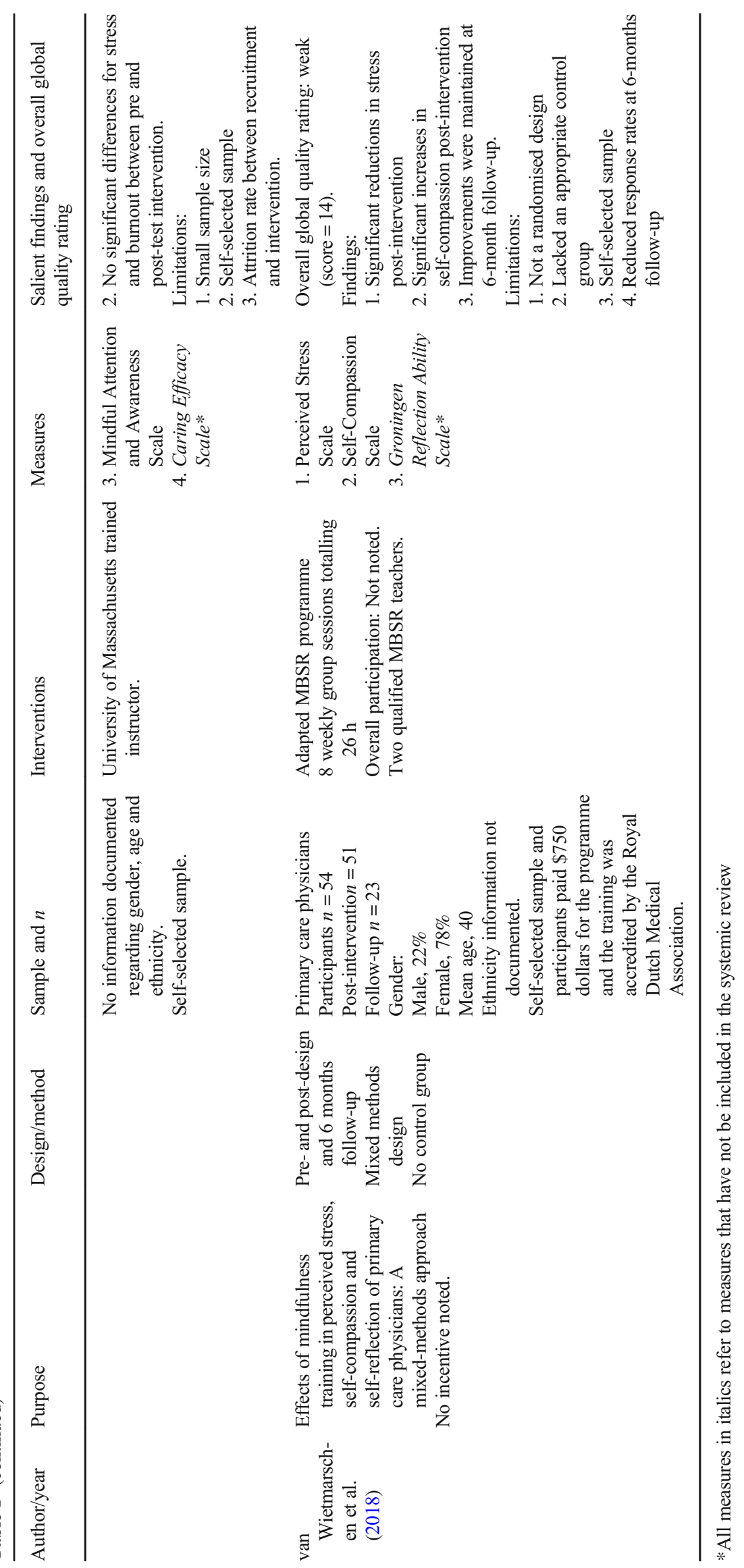


stress and leadership strategies course and another study had a control group which attended a humanities class. No studies included a treatment comparison group. Thirteen studies collected follow-up data, ranging from 2 months to 1 year.

\section{Study Populations and Settings}

Participant numbers ranged from a minimum of twelve to a maximum of ninety-three and the total number of participants (final $n$ ) across all thirty studies included in the review was 1053. Twenty-six studies included gender information of participants. With the exception of three studies (Ducar et al. 2019; Pflugeisen et al. 2016; Verweij et al. 2016), there was an overrepresentation of females within all the study samples compared with males. Twenty-three studies documented participant age information, and the mean age ranges varied from 22.7 to 52.2 years.

Four studies detailed ethnicity information of participants, and there was an overrepresentation of Caucasian participants in three studies (Crowder and Sears 2017; Fortney et al. 2013; Geary and Rosenthal 2011) and an overrepresentation of Chinese participants in another study (Suyi et al. 2017). Sixteen of the studies were carried out in America, three in Spain, two in Portugal, two in the Netherlands, two in Canada, one in Australia, one in Japan, one in Singapore, one in Taiwan and one in Iran.

With regard to the HCP disciplines, seven studies focused on nursing staff, five studies focused on physicians, two studies focused on social workers, one study on public health workers and two studies on voluntary workers, namely emergency medical staff and workers supporting women with breast cancer. The remaining twenty-three studies focused on a variety of HCPs including all the aforementioned disciplines. Additional HCPs included physical therapists, respiratory therapists, activity therapists, occupational therapists, support staff, pharmacists, researchers, a dentist and a nutritionist. All participants were self-selected, and within four studies (Amutio et al. 2015; Goodman and Schorling 2012; van Wietmarschen et al. 2018; Verweij et al. 2016), participants voluntarily took part in the MBSR courses as part of a group for the purpose of continual professional development (CPD).

\section{MBSR Programme Characteristics}

All MBSR programmes were facilitated face to face and in group format, with the exception of two studies. One study (Bazarko et al. 2013) used a group telephonic (tMBSR) format, which involved two 8-h retreats and six 90-min group teleconference calls, with email contact in-between sessions. Another study (Pflugeisen et al. 2016) facilitated an 8-week MBSR programme with three $90 \mathrm{~min}$ in person training sessions, with five weekly online 7-min video training modules, along with 1 -h weekly teleconference coaching calls. The remaining studies included standard MBSR programmes, abbreviated or modified MBSR programmes. With regard to programme duration, programmes varied from 2 days to 11 weeks and one programme had a 10-month maintenance phase.

\section{Outcome Measures}

\section{Stress}

Eighteen studies reported significant reductions in stress and four studies reported non-significant findings (Duchemin et al. 2015; Martin-Asuero and Garcia-Banda 2010; Moody et al. 2013; Trowbridge et al. 2017). Of note, one study reported a reduction in stress on the Depression Anxiety Stress Scale (Lovibond and Lovibond 1995), but a non-significant finding on the Perceived Stress Scale (Cohen et al. 1983; Duchemin et al. 2015). Significant reductions in stress were also maintained ranging from 2 months (Hallman et al. 2017) to 1-year (Geary and Rosenthal 2011) follow-up. However, significant reductions in stress were not maintained at 3-month follow-up (Ducar et al. 2019; Suyi et al. 2017) and 6-month follow-up (Ducar et al. 2019).

\section{Burnout}

With regard to burnout, thirteen studies reported significant reductions in burnout. However, nine studies found nonsignificant findings for burnout. Significant reductions in burnout were maintained ranging from 3-month (Ducar et al. 2019; Schroeder et al. 2016) to 9-month (Fortney et al. 2013) follow-up. One study found that reductions in burnout were not sustained at 3-month follow-up (Ceravolo and Raines 2019). Of note, Crowder and Sears (2017) reported a nonsignificant finding for burnout at 13-week follow-up; however, a significant reduction in burnout was later reported at 26week follow-up.

\section{Mood}

In relation to mood disturbance, one study reported a significant improvement in mood disturbance, five studies reported significant reductions in anxiety, four studies found significant reductions in depression, one study reported significant improvements in positive affect and two studies reported significant reductions in negative affect. However, two studies reported non-significant findings in anxiety (Duarte and Pinto-Gouveia 2016; Duchemin et al. 2015), four studies found non-significant findings in depression (Dobie et al. 2016; Duarte and Pinto-Gouveia 2016; Duchemin et al. 2015; Moody et al. 2013), and a further study reported nonsignificant changes in positive and negative affect (Cucarella 
and Gianinni 2016). Significant reductions in anxiety, depression and stress were maintained at 9-month follow-up (Fortney et al. 2013).

\section{Psychological Distress}

For measures of psychological distress and mental wellbeing, seven studies reported significant improvements in psychological distress, mental wellbeing, and mental health and one study reported a non-significant finding for improvements in wellness (Ceravolo and Raines 2019). Significant improvements in psychological distress were maintained at 3-month (Martin-Asuero and Garcia-Banda 2010) and 1-year follow-up (Geary and Rosenthal 2011; compared to controls), and significant improvements of mental wellbeing were also reported at one-year followup compared to controls (Geary and Rosenthal 2011). Significant improvements in mental health were also maintained at 4-month follow-up (Bazarko et al. 2013).

\section{Self-Compassion and Resilience}

With regard to measures of self-compassion, eight studies reported significant increases in self-compassion. Significant improvements in self-compassion were also maintained and ranged from 3-months (Suyi et al. 2017) to 26-week follow-up (Crowder and Sears 2017). For measures of resilience, only two studies measured resilience and both reported nonsignificant findings (Fortney et al. 2013; Schroeder et al. 2016).

\section{Mindfulness}

In relation to measures of mindfulness, fifteen studies reported significant improvements in overall mindfulness and in all facets of mindfulness (observe, describe, acting with awareness and non-judging) with the exception of nonreacting. However, non-significant findings were also reported for the facet observe (Dobie et al. 2016), describe (Manotas et al. 2014; Martin-Asuero et al. 2014), acting with awareness and non-reactiveness (Manotas et al. 2014). In terms of follow-up, significant improvements in mindfulness were observed ranging from 2 months (Hallman et al. 2017) to 3 months (Brady et al. 2012; Ducar et al. 2019; Schroeder et al. 2016; Suyi et al. 2017). However, in several studies significant improvements in mindfulness were not maintained at 3 (Wang et al. 2017) or 6 months (Ducar et al. 2019; Wang et al. 2017) follow-up.

\section{Discussion}

The systematic review findings provided further evidence that MBSR programmes were effective in improving particular aspects of psychological functioning in different types of HCPs, including reducing anxiety, depression, and stress and increasing self-compassion. These findings supported theories that mindfulness can generate a positive shift in perspective and an ability to view one's life experiences more objectively. This supported Shapiro et al.'s (2006) theory that mindfulness has three mechanisms (intention, attention and attitude) and Bishop et al.'s (2004) two-component model of mindfulness. Shapiro et al. (2006) and Bishop et al. (2004) postulated that improvements in self-regulation and nonreactive awareness could lead to changes in perspective. This process of decentering (Baer 2003; Brown et al. 2007) or reperceiving (Shapiro et al. 2006) leads individuals to employ a 'mindfulness-mediated stress response' (Kabat-Zinn 2013) and thus be less affected by the power of the stress response. With the development of such skills, HCPs would be expected to display improved stress management and reduced levels of stress and burnout.

However, the systematic review also highlighted that MBSR were less effective in reducing burnout and increasing resilience, compared with other aspects of psychological functioning. The inconsistent findings could be explained by research that has previously suggested that burnout is a distinct construct, which is connected to mental health difficulties such as anxiety, depression and stress (Awa et al. 2010; Maslach et al. 2001), but is not the same. Levels of burnout may take longer to decrease compared with mental health difficulties, and it is only following the implementation of healthier coping strategies, such as decentering and reperceiving, that levels of burnout may reduce. This interpretation seems to be supported by Crowder and Sears (2017), who reported non-significant findings for burnout at 13-week follow-up for an MBSR programme for social workers, but later found significant reductions at 26-week follow-up. Arguably, resilience is a trait that develops over time and may be less amenable to change following an MBSR intervention compared to other emotional states such as anxiety, depression and stress. Further research is therefore required into the underlying relationships between burnout, resilience and mindfulness, which in turn may provide greater insight into how HCPs burnout and resilience can be targeted using effective MBIs.

Findings from the systematic review also suggested that MBSR programmes were effective in increasing HCPs overall levels of mindfulness. However, when specific facets of mindfulness were investigated, MBSR appeared not to have consistent positive effects. The varied findings could be attributed to the HCPs developing some aspects of mindfulness skills associated with anxiety and stress during the completion of the MBSR programme. Alternatively, like resilience some facets of mindfulness may take longer to develop, such as acting with awareness and non-reactiveness. Furthermore, fourteen studies in the review did not measure mindfulness as an 
outcome measure pre- and post-intervention, thus making it difficult to ascertain whether effective treatment outcomes were a consequence of increased mindfulness skills (Spinelli et al. 2019) or other factors such as social support of group cohesion. Further research is required to explore whether the facets of mindfulness improve with training and to identify how MBSR programmes can be adapted to target all of the facets of mindfulness. Future research into the underlying mechanisms of mindfulness and how these may play a role in producing the positive treatment outcomes that have been observed in MBSR effectiveness studies is also recommended.

The robustness of the available research included in the systematic review indicated that the overall quality proved weak to moderate and that methodological limitations were common (e.g. variability in methodology, outcome measures, small sample sizes, self-selected samples and overrepresentation of Caucasian women). Many of the studies lacked randomisation, blinding procedures and control groups, and none of the four RCTs included an alternative treatment comparison. All studies obtained a weak rating for selection bias, as all samples were self-selected. Selection bias may have influenced the results of non-randomised studies, as randomly assigning participants to either control or experimental group is critical in reducing selection bias. Due to the samples being self-selected, only motivated participants would have arguably completed the MBIs and thus would be more likely to report positive treatment outcomes (Klein et al. 2019). This was particularly relevant to four research studies whereby participants completed MBSR courses on a voluntary basis as part of their CPD and within two studies participants paid for the intervention (Goodman and Schorling 2012; van Wietmarschen et al. 2018).

In addition, the participant samples were noted to be overrepresentative of women compared to men in the studies, thus reducing the generalising of study findings. Twenty-six of the studies did not report ethnicity participant information. Within four studies that detailed ethnicity information, there was an overrepresentation of Caucasian and Chinese participants, again limiting the generalisability of findings. With regard to confounding variables, the studies that had a control group or waitlist control generally did explore preexisting differences between groups regarding demographic characteristics and pre-intervention measure scores. If significant differences were found between groups, statistical analyses were generally used to control such differences. Future studies are encouraged to employ robust controlled designs with 'active' control groups (Lomas et al. 2019), obtain larger sample sizes and more heterogeneous samples to increase the generalisability of research findings.

Unfortunately, some studies did not assess for previous experience of mindfulness practice which may have influenced the outcome of the research findings. With regard to measuring mindfulness home practice, research suggested that participants, who completed mindfulness home practice along with completing an MBSR programme, were more likely to observe greater improvements than participants who did not (Carmody and Baer 2009). Thus, exploring the frequency and intensity of mindfulness practice external to MBSR programmes proved very important, along with assessing its influence upon treatment outcomes (Spinelli et al. 2019). Fourteen studies encouraged participants to engage in daily formal mindfulness, which ranged from 15 to $45 \mathrm{~min}$, throughout the duration of the MBSR programmes. Sixteen studies did not include information about formal daily mindfulness practice and six studies monitored participants mindfulness practice.

Moody et al. (2013) found that participants practiced at least one mindfulness technique eight to ten times per week. Interestingly, MBSR did not result in any consistent or significant improvements in burnout, stress or depression. Moody et al. (2013) argued that their sample of paediatric and oncology staff had significantly higher levels of burnout and stress compared with other studies where MBSR had demonstrated a positive effect with HCPs (Cohen-Katz et al. 2005; Shapiro et al. 2005). In contrast, Bazarko et al. (2013) found that nurses, that maintained the mindfulness practice compared to those that did not, reported significantly lower stress and burnout scores. Whilst Duarte and Pinto-Gouveia (2016) reported that oncology nurses that practiced more mindfulness showed greater reductions in burnout and depression, and greater increases in self-compassion compared to those that practiced less. Ruiz-Fernandez et al. (2019) postulated that when daily mindfulness practice was encouraged, stress levels reduced and mindfulness increased.

Overall, the findings from the systematic review suggested that mindfulness home practice may lead to observed improvements in some aspects of psychological functioning amongst HCPs. Therefore, the design of future MBIs should incorporate daily meditation practice to maximise the effectiveness of the intervention. Briefer mindfulness practices may help increase the level of mindfulness home practice. Further research into the quality and quantity of formal and informal mindfulness home practice may help achieve psychological health outcomes (Irving et al. 2009).

Some studies employed the traditional 8-week MBSR programme and other studies utilised abbreviated MBSR programmes. With regard to the duration of the reviewed MBSR programmes, eighteen programmes were 8 weeks in duration. Eight of which followed the traditional MBSR model, namely eight 2.5 -h weekly sessions along with a day retreat. One of these MBSR programmes also had a 10-month maintenance phase that involved one session of $2.5 \mathrm{~h}$ per month. Two MBSR programmes were 6 weeks in duration and yielded significant improvements in stress, burnout, mindfulness and self-compassion (Duarte and Pinto-Gouveia 
2016; Suyi et al. 2017) and in one study improvements in mindfulness and self-compassion were maintained at 3month follow-up. Three MBSR programmes were 4 weeks in duration and yielded significant improvements in psychological distress, anxiety, depression, stress and mindfulness (Brady et al. 2012; Pipe et al. 2009; Verweij et al. 2016) and in one study the improvements in stress and mindfulness were sustained at 3-month follow-up (Brady et al. 2012) although the results for burnout proved non-significant.

Three MBSR programmes were less than 3 weeks in duration and yielded significant reductions in anxiety, depression, stress and burnout and significant improvements in mindfulness (Ando et al. 2011; Fortney et al. 2013, Hallman et al. 2017). Improvements in stress and burnout were maintained at 2-month follow-up (Hallman et al. 2017) and improvements in anxiety, depression, stress and burnout were maintained at 9-month follow-up (Fortney et al. 2013). Findings were nonsignificant for resilience (Fortney et al. 2013). Two MBSR programmes were 2 days in duration and yielded significant improvements for stress, mindfulness, emotional exhaustion and depersonalisation (Schroeder et al. 2016; Trowbridge et al. 2017) and were maintained at 3-month follow-up. However, non-significant findings for stress, burnout (Trowbridge et al. 2017), self-compassion, resilience and personal accomplishment (Schroeder et al. 2016) were also reported. Another programme was 11 weeks in duration. For one of the programmes, there was no information noted about the duration of the programme.

In line with previous research (MacKenzie et al. 2006; Shapiro et al. 2005; Virgili 2013), the systematic review highlighted that abbreviated MBSR programmes (e.g. 4 to 7 weeks) proved as effective as traditional MBSR programmes. Even MBSR programmes that were shortened and delivered within a 3-week period, produced effective treatment outcomes for (1) stress and mindfulness maintained at 2-month follow-up (Hallman et al. 2017) and (2) anxiety, depression, stress and burnout maintained at 9-month followup (Fortney et al. 2013). Overall, the findings suggested that brief MBSR programmes of 3 weeks (or less) were effective in improving the psychological functioning of HCPs. In addition, future research determining which components of MBSR are driving the effects is warranted, such as exercises that focus more on developing decentering skills. This would help design the content of future MBIs for HCPs and help maximise their effectiveness.

The findings also highlighted that uncertainty remains regarding the optimal amount of MBSR needed to alleviate stress and other emotional constructs (Smith 2014). The findings may prove helpful when considering how to adapt and implement MBSR programmes for HCPs, in particular those facilitated within inpatient settings, where attendance may prove increasingly challenging due to staffing levels. Furthermore, shorter MBSR programmes cost significantly less to deliver, which is an important factor in the current climate where healthcare organisations are under constant pressure to make financial savings. Further research to identify the optimal adapted MBSR programme design and how this may affect treatment outcomes for HCPs is warranted.

With regard to overall participant attendance, eighteen studies did not report data for overall participant attendance. In relation to the remaining twelve studies, participant attendance rates were reported in varying ways, thus making it difficult to reliably compare the data. Although some studies reported high overall attendance, it is important to note that these numbers may not include busy and stressed HCPs from participating. With regard to participant attendance, all studies in the systematic review included attrition data with the exception of three studies (Ando et al. 2011; Cucarella and Gianinni 2016; Norouzinia et al. 2017). Six studies had no dropouts or withdrawals, and in the remaining twenty-one studies, the attrition rates varied from 3 to $57 \%$. Although no incentives were noted in twenty-one of the studies, three studies paid participants directly for their involvement and the remaining six studies gave participants a monetary incentive, education credits and/or a coupon following each completion of questionnaires or study visit. Two MBSR programmes were part of a regular CPD programme accredited by professional bodies and in another study, participants were paid a portion of their work time. Arguably studies that included incentives for participation may have positively biased their results with regard to attrition rates or possibly outcomes (Bazarko et al. 2013). Further research is recommended to explore how the provision of incentives for participation in MBSR programmes effects attrition rates and treatment outcomes.

Three studies carried out MBSR programmes in psychiatric inpatient units. In the first study, HCPs were allocated 15-min meditation time within their working day (Hallman et al. 2017). In Brady et al.'s (2012) study, HCPs were paid for attending MBSR classes, and in Dobie et al.'s (2016) research, mindfulness sessions were facilitated in the morning at the beginning of shifts. However, Hallman et al. (2017) found that only a few HCPs used the allocated meditation time and Brady et al. (2012) reported a $30 \%$ attrition rate due to the varying work schedule demands of the unit. Bryon et al. (2015) identified several barriers to the implementation of mindfulness training for HCPs working within psychiatric inpatient units, which included limited time for staff to attend training sessions, insufficient cover to allow staff to participate in the sessions without using personal time and inadequate preparation for the new initiative on some units. Due to the practical difficulties and time restrictions often faced by HCPs, shorter MBSR programmes incorporated into the regular working schedules of staff have been recommended to reduce attrition rates (Shapiro et al. 2007). Hallman et al. (2017) further argued that the shorter duration of the MBSR 
intervention (e.g. 8 days versus 8 weeks) in their study may have accounted for the retention of participants at 2-month follow-up. Overall, the findings suggested that shorter, wellorganised MBSR programmes embedded into the working schedules of HCPs could reduce attrition rates and increase engagement in MBIs.

Despite some research studies employing robust outcome measures, great variability was found between studies that investigated different aspects of psychological functioning (e.g. anxiety, depression, stress, burnout and mindfulness). Such variability could lead to ambiguity and difficulty comparing findings between studies that investigate the same psychological construct. Future research would benefit from employing consistent assessments to measure outcomes of psychological functioning (Escuriex and Labbe 2011; Smith 2014), so that findings between studies can be compared reliably and allow defensible conclusions to be made.

In relation to programme integrity, Irving et al. (2009) argued that further investigation into the integrity of MBSR programmes was pertinent. Of the thirty studies included in the systematic review, twenty-four studies employed certified MBSR instructors and mindfulness teachers and six studies failed to include any information about the MBSR facilitators. Of note, four of the studies MBSR facilitators were also part of the research team, thus potentially reinforcing selection bias due to social desirability effects.

\section{Future Research}

The systematic review highlighted that MBSR was effective in reducing HCPs experiences of anxiety, depression and stress. MBSR was also found to be effective in increasing HCP levels of mindfulness and self-compassion. However, MBSR did not appear as effective in reducing burnout or improving resilience amongst HCPs. Arguably burnout and resilience may be conceptualised as different constructs to depression, anxiety and stress. Burnout and resilience may be viewed as traits rather than states, thus potentially taking longer to improve, requiring the longer-term implementation of healthier coping strategies which then facilitate improvements in burnout and resilience. The review also provided further support for existing mindfulness theories that have highlighted the importance of decentering and reperceiving as essential mechanisms in reducing HCPs vulnerability to the impact of the stress response. Further research exploring the underlying mechanisms that maximise the effectiveness of MBSR interventions on different psychological constructs would be of great benefit to the current evidence base.

Furthermore, the review highlighted that abbreviated MBSR programmes were as effective as traditional 8-week MBSR programmes and that briefer, well-organised programmes embedded into HCP working days proved more successful. The review also highlighted the importance of future MBSR programmes encouraging and monitoring daily mindfulness practice to maximise treatment outcome. Existing studies were generally found to be of poor quality with shortcomings related to self-selected samples, lack of controlled studies and randomisation, lack of blinding and overrepresentation of Caucasian women within samples. Future studies need to incorporate robust study designs (e.g. RCTs), consistent outcome measures, larger sample sizes, heterogeneous samples and compare MBSR programmes with other active controls or psychological interventions designed to improve psychological functioning amongst HCPs. Future studies would benefit from longer-term follow-up to facilitate exploration of the continuing effects of mindfulness on different psychological constructs. Such research could help facilitate the development of a relevant psychological model for the underlying mechanisms of mindfulness and its relationship with other psychological outcomes, such as stress, burnout and resilience.

Author Contributions SAK designed and executed the systematic review and wrote the paper. KAE collaborated with the design and writing of the systematic review and editing of the final manuscript. CL collaborated with the design and execution of the systematic review and editing of the final manuscript. RO collaborated with the design of the systematic review.

\section{Compliance with Ethical Standards}

Conflict of Interest The authors declare they have no conflict of interest.

Ethical Approval This article does not contain any studies with human participants performed by any of the authors.

\section{References}

* Indicates studies included in the systematic review

*Amutio, A., Martinez-Taboada, C., Delgado, L.C., Hermosilla, D., \& Mozaz, M.J. (2015). Acceptability and effectiveness of a long-term educational intervention to reduce physicians stress-related conditions. Journal of Continuing Education in the Health Professions, 35 (4), 255-260. https://doi.org/10.1097/ceh.0000000000000002.

*Ando, M., Natsume, T., Kukihara, H., Shibata, H., \& Ito, S. (2011). Efficacy of mindfulness-based meditation therapy on the sense of coherence and mental health of nurses. Health, 3 (2), 108-122. https://doi.org/10.4236/health.2011.32022.

Awa, W. L., Plaumann, M., \& Walter, U. (2010). Burnout prevention: A review of intervention programs. Patient Education and Counselling, 78(2), 184-190. https://doi.org/10.1016/j.pec.2009. 04.008 .

Baer, R. A. (2003). Mindfulness training as a clinical intervention: A conceptual and empirical review. Clinical Psychology: Science and Practice, 10(2), 125-143. https://doi.org/10.1093/clipsy. bpg015.

*Bazarko, D., Cate, R.A., Azocar, F., \& Kreitzer, M.J. (2013). The impact of innovative mindfulness-based stress reduction program on the health and well-being of nurses employed in a corporate setting. 
Journal of Workplace Behavioral Health, 28 (2), 107-133. https:// doi.org/10.1080/15555240.2013.779518.

Bishop, S. R., Lau, M., Shapiro, S., et al. (2004). Mindfulness: A proposed operational definition. Clinical Psychology: Science and Practice, 11, 230-241. https://doi.org/10.1093/clipsy.bph077.

*Brady, S., O’Connor, N., Burgermeister D., \& Hanson, P. (2012). The impact of mindfulness meditation in promoting a culture of safety on an acute psychiatric unit. Perspectives in Psychiatric Care, 48, 129137. https://doi.org/10.1111/j.1744-6163.2011.00315.x.

Brown, K. W., Ryan, R. M., \& Creswell, J. D. (2007). Mindfulness: Theoretical foundations and evidence for its salutary effects. Psychological Inquiry, 18(4), 211-237. https://doi.org/10.1111/j. 1744-6163.2011.00315.x.

Bryon, G., Ziedonis, D. M., McGarth, C., Frazier, J. A., deTorrijos, F., \& Fulwiler, C. (2015). Implementation of mindfulness training for mental health staff: Organisational context and stakeholder perspectives. Mindfulness, 6, 861-872. https://doi.org/10.1007/s12671014-0330-2.

Burton, A., Burgess, C., Dean, S., Koutsopoulou, G. Z., \& Hugh-Jones, S. (2017). How effective are mindfulness-based interventions for reducing stress among healthcare professionals? A systemic review and meta-analysis. Stress and Health, 33, 3-13. https://doi.org/10. 1002/smi.2673.

Byrant, C., Fairbrother, G., \& Fenton. (2000). The reflective influence of personal and workplace descriptors of stress. British Journal of Nursing, 9(13), 876-880. https://doi.org/10.12968/bjon.2000.9.13. 5517.

Carmody, J., \& Baer, R. A. (2009). How long does mindfulness-based stress reduction program need to be? A review of class contact hours and effect sizes for psychological distress. Journal of Clinical Psychology, 65(6), 627-638. https://doi.org/10.1002/jclp.20555.

*Ceravolo, D. \& Raines, D.A. (2019). The impact of a mindfulness intervention for nurse managers. Journal of Holistic Nursing, 37 (1), 47-55. https://doi.org/10.1177/0898010118781620.

Chiesa, A., \& Serretti, A. (2009). Mindfulness-based stress reduction for stress management in healthy people: A review and meta-analysis. The Journal of Alternative and Complementary Medicine, 15(5), 593-600. https://doi.org/10.1089/acm.2008.0495.

Cohen, S., Kamarck, T., \& Mermelstein, R. (1983). A global measure of perceived stress. Journal of Health and Social Behaviour, 24(4), 385-396. https://doi.org/10.2307/2136404.

Cohen-Katz, J. C., Wiley, S. D., Capuano, T., Baker, M. A., \& Shapiro, S. (2005). The effects of mindfulness-based stress reduction on nurse stress and burnout, part II: A quantitative and qualitative study. Holistic Nursing Practice, 19, 26-35. https://doi.org/10.1097/ 00004650-200501000-00008.

*Crowder, R. \& Sears, A. (2017). Building resilience in social workers: An exploratory study on the impacts of a mindfulness-based intervention. Australian Social Work, 70(1), 17-29. https://doi.org/10. 1080/0312407x.2016.1203965.

*Cucarella, S.P. \& Gianinni, M.C. (2016). Effectiveness of a pilot mindfulness program in volunteers for a breast cancer association. Journal of Evidence-Based Complementary and Alternative Medicine, 21(4), 291-294. https://doi.org/10.1177/ 2156587215602408.

*Dobie, A., Tucker, A., Ferrari, M., \& Rogers, J.M. (2016). Preliminary evaluation of a brief mindfulness-based stress intervention for mental health professionals. Australasian Psychiatry, 24(1), 42-45. https://doi.org/10.1177/1039856215618524.

Dougherty, E., Pierce, B., Ma, C., Panzarella, T., Rodin, G., \& Zimmermann, C. (2009). Factors associated with work stress and professional satisfaction in oncology staff. American Journal of Hospice and Palliative Medicine, 26, 105-111. https://doi.org/10. 1177/1049909108330027.

*Duarte, J. \& Pinto-Gouveia, J. (2016). Effectiveness of a mindfulnessbased intervention on oncology nurses' burnout and compassion fatigue symptoms: A non-randomized study. International Journal of Nursing Studies, 64, 98-107. https://doi.org/10.1016/j.ijnurstu. 2016.10.002.

*Ducar, D.M., Penberthy, J.M., Schorling, J.B., Leavell, V.A., \& Calland, J.F. (2019). Mindfulness for healthcare providers fosters professional quality of life and mindful attention among emergency medical technician. Explore, 16 (1), 61-68. https://doi.org/10.1016/ j.explore.2019.07.015.

*Duchemin, A.M., Steinberg, B.A., Marks, D.R., Vanover, K., \& Klatt, M. (2015). A small randomized pilot study of a workplace mindfulness-based intervention for surgical intensive care unit personnel: Effects on salivary amylase levels. Journal of Occupational and Environmental Medicine, 57(4); 393-399. https://doi.org/10. 1097/jom.0000000000000371.

Effective Public Health Practice Project (EPHPP). (1998). Quality assessment tool for quantitative studies. Hamilton, ON: Effective Public Health Practice Project. Retrieved from: http://www.ephpp.ca/ index.html. Accessed 01.12.2014.

Escuriex, B. F., \& Labbe, E. E. (2011). Healthcare providers' mindfulness and treatment outcomes: A critical review of the research literature. Mindfulness, 2, 242-253. https://doi.org/10.1007/s12671011-0068-z.

Fahrenkopf, A. M., Sectish, T. C., Barger, L. K., Sharek, P. J., Lewin, D., Chiang, V. W., et al. (2008). Rates of medication errors among depressed and burnt out residents: A prospective cohort study. British Medical Journal, 336, 488-491. https://doi.org/10.1136/ bmj.39469.763218.be.

Farber, B. A. (1983). Introduction: A critical perspective on burnout. In B. A. Farber (Ed.), Stress and burnout in the human services professions (pp. 1-20). New York, NY: Pergamon.

*Fortney, L., Luchterhand, C., Zakletskaia, L., Zgierska, A., \& Rakel, D. (2013). Abbreviated mindfulness intervention for job satisfaction, quality of life, and com passion in primary care clinicians: A pilot study. Annals of Family Medicine, 11(5), 412-420. https://doi.org/ 10.1370/afm.1511.

*Gaspar, I., Martinho, A., \& Lima, M. (2018). Exploring the benefits of a mindfulness program for Portuguese public healthcare workers. Current Psychology. https://doi.org/10.1007/s12144-018-9987-3.

*Geary, C. \& Rosenthal, S. (2011). Sustained impact of MBSR on stress, well-being, and daily spiritual experiences for one year in academic healthcare employees. The Journal of Alternative and Complementary Medicine, 17(10), 939-944. https://doi.org/10. 1089/acm.2010.0335.

*Goodman, M. J. \& Schorling, J.B. (2012). A mindfulness course decreases burnout and improves well-being among healthcare providers. International Journal of Psychiatry in Medicine, 43(2), 119-128. https://doi.org/10.2190/pm.43.2.b.

Gu, J., Strauss, C., Bond, R., \& Cavanagh, K. (2015). How do mindfulness-based cognitive therapy and mindfulness-based stress reduction improve mental health and well-being? A systematic review and meta-analysis of mediation studies. Clinical Psychology Review, 37, 1-12. https://doi.org/10.1016/j.cpr.2016.09.011.

*Hallman, I.S., O’Connor, N., Hasenau, S., \& Brady, S. (2017). Improving the culture of safety on a high-acuity inpatient child/ adolescent psychiatric unit by mindfulness-based stress reduction training of staff. Journal of Child and Adolescent Psychiatric Nursing, 30, 175-180. https://doi.org/10.1111/jcap.12191.

Hayes, S. C., \& Wilson, K. G. (1994). Acceptance and commitment therapy: Altering the verbal support for experiential avoidance. The Behaviour Analyst, 17, 289-303. https://doi.org/10.1007/ bf03392677.

Irving, J. A., Dobkin, P. L., \& Park, J. (2009). Cultivating mindfulness in healthcare professionals: A review of empirical studies of mindfulness-based stress reduction (MBSR). Complementary Therapies in Clinical Practice, 15, 61-66. https://doi.org/10.1016/ j.ctcp.2009.01.002. 
Kabat-Zinn, J. (1982). An outpatient program in behavioral medicine for chronic pain patients based on the practice of mindfulness meditation: Theoretical considerations and preliminary results. General Hospital Psychiatry, 4(1), 33-47. https://doi.org/10.1016/01638343(82)90026-3

Kabat-Zinn, J. (1994). Wherever you go, there you are: Mindfulness meditation in everyday life. New York, NY: Hyperion Books.

Kabat-Zinn, J. (2013). Full catastrophe living: Using the wisdom of your body and mind to face stress, pain, and illness. New York, NY: Delta.

Klein, A., Taieb, O., Xavier, S., Baubet, T., \& Reyre, A. (2019). The benefits of mindfulness-based interventions on burnout among health professionals: A systematic review. Explore, 16(1), 35-43. https://doi.org/10.1016/j.explore.2019.09.002.

Lazarus, R. S., \& Folkman, S. (1984). Stress, appraisal and coping. New York, NY: Springer.

Lomas, T., Medina, J. C., Ivtzan, I., Rupprecht, S., \& Eiroa-Orosa, F. F. (2019). A systematic review and meta-analysis of the impact of mindfulness-based interventions on the well-being of healthcare professionals. Mindfulness, 10, 1193-1216. https://doi.org/10. 1007/s12671-018-1062-5.

Lovibond, S. H., \& Lovibond, P. F. (1995). Manual for the depression anxiety stress scales (2nd ed.). Sydney, Australia: Psychology Foundation

MacKenzie, C. S., Poulin, P. A., \& Seidman-Carlson, R. A. (2006). A brief-mindfulness based stress reduction intervention for nurses and nurse aides. Nursing Research, 19, 105-109. https://doi.org/10. 1016/j.apnr.2005.08.002.

*Manotas, M., Segura, C., Eraso, M., Oggins, J., \& McGovern, K. (2014). Association of brief mindfulness training with reductions in perceived stress and distress in Colombian healthcare professionals. International Journal of Stress Management, 21(2), 207225. https://doi.org/10.1037/a0035150.

*Martin-Asuero, A. \& Garcia-Banda, B. (2010). The mindfulness-based stress reduction program (MBSR) reduces stress-related psychological distress in healthcare professionals. The Spanish Journal of Psychology, 13(2), 897-905. https://doi.org/10.1017/ s1138741600002547.

*Martin-Asuero, A., Queralto, J.M., Pujol-Ribera, E., Berenguera, A., Rodriguez-Blanco, T., \& Epstein, R.M. (2014). Effectiveness of a mindfulness education program in primary health care professionals: A pragmatic controlled trial. Journal of Continuing Education in the Health Professions, 34(1): 4-12. https://doi.org/ 10.1002/chp.21211.

Maslach, C., Jackson, S. E., \& Leiter, M. P. (1996). Maslach burnout inventory manual (3rd ed.). Palo Alto: Consulting Psychologists Press, Inc..

Maslach, C., Schaufeli, W. B., \& Leiter, M. P. (2001). Job burnout. Annual Review of Psychology, 52, 397-422. https://doi.org/10. 1146/annurev.psych.52.1.397.

Miller, K. I., Stiff, J. B., \& Ellis, B. H. (1988). Communication and empathy as precursors to burnout among human service workers. Communication Monographs, 55, 250-265. https://doi.org/10. 1080/03637758809376171.

Moher, D., Liberati, A., Tetzlaff, J., \& Altman, D. G. (2009). Preferred reporting terms for systematic reviews and meta-analyses: The PRISMA statement. PLoS Medicine, 6(7), 1-6. https://doi.org/10. 1371/journal.pmed.1000097.

*Moody, K., Kramer, D., Santizo, R.O., Magro, L., Wyshogrod, D., Ambrosio, J., Castillo, C., Lieberman, R., \& Stein, J. (2013). Helping the helpers: Mindfulness training for burnout in paediatric oncology - a pilot program. Journal of Paediatric Oncology Nursing, 30(5), 275-284. https://doi.org/10.1177/ 1043454213504497.
Morgan, P., Simpson, J., \& Smith, A. (2014). Healthcare workers' experiences of mindfulness training: A qualitative review. Mindfulness, 6, 744-758. https://doi.org/10.1007/s12671-014-0313-3.

National Collaborating Centre for Methods and Tools. (2008). Quality assessment tool for quantitative studies. Hamilton, ON: McMaster University. http://www.nccmt.ca/registry/view/eng/14. html Accessed 01.12.2014.

*Norouzinia, R., Ramezani, Z., Khalili, A., Dehghani, M., \& Sharifi, A. (2017). The effect of mindfulness-based stress reduction training on stress and burnout of nurses. Indo American Journal of Pharmaceutical Sciences, 4(5), 1296-1302. http://www.iajps.com/ pdf/may2017/34.IAJPS34052017.pdf. Accessed 10.04.2020.

*Pflugeisen, B.M., Drummond, D., Ebersole, D., Mundell, K., \& Chen, D. (2016). Brief video-module administered mindfulness program for physicians: A pilot study. Explore, 12 (1), 50-54. https://doi.org/ 10.1016/j.explore.2015.10.005.

*Pipe, T. B., Bortz, J.J., \& Duek, A. (2009). Nurse leader mindfulness meditation program for stress management: A randomized controlled trial. The Journal of Nursing Administration, 39(3), 130 137. https://doi.org/10.1097/nna.0b013e31819894a0.

Pipe, T., Fitzpatrick, K., Doucette, J. N., Cotton, A., \& Arnow, D. (2016). The mindful nurse leader: Improving processes and outcomes; restoring joy to nursing. Nursing Management, 47(9), 44-48. https:// doi.org/10.1097/01.numa.0000491135.83601.3e.

Preedy, V. R., \& Watson, R. R. (2010). Handbook of disease burdens and quality of life measures. New York, NY: Springer-Verlag.

*Raab, K., Sogge, K., Parker, N., \& Flament, M.F. (2015). Mindfulnessbased stress reduction and self-compassion among mental healthcare professionals: A pilot study. Mental Health, Religion and Culture, 18(6), 503-512. https://doi.org/10.1080/13674676. 2015.1081588.

Ruiz-Fernandez, M. D., Artiz-Amo, R., Ortega-Galan, A. M., IbanezMasero, O., Rodriguez-Salvador, M. D., \& Ramos-Pichardo, J. D. (2019). Mindfulness therapies on health professionals. International Journal of Mental Health Nursing, 29(2), 127-140. https://doi.org/ 10.1111/inm. 12652

Rupert, P. A., \& Morgan, D. J. (2005). Work setting and burnout among professional psychologists. Professional Psychology: Research and Practice, 36, 544-550. https://doi.org/10.1037/0735-7028.36.5. 544.

Schinler, B. A., Novack, D. H., Cohen, D. G., Yager, J., Wang, D., Saheen, N. J., et al. (2006). The impact of the changing care environment on the health and well-being of faculty at four medical schools. Academic Medicine, 81, 27-34. https://doi.org/10.1097/ 00001888-200601000-00008.

*Schroeder, D.A., Stephens, E., Colgan, D., Hunsinger, M., Rubin, D., \& Christopher, M.S. (2016). A brief mindfulness-based intervention for primary care physicians: A pilot randomized controlled trial. American Journal of Lifestyle Medicine, 12(1): 83-91. https://doi. org/10.1177/1559827616629121.

Schulz, R., Greenley, J. R., \& Brown, R. (1995). Organisation, management, and client effects on staff burnout. Journal of Health and Social Behaviour, 36(4), 333-345. https://doi.org/10.2307/ 2137323.

Segal, Z. V., Williams, M. G., \& Teasdale, J. D. (2002). Mindfulnessbased cognitive therapy for depression: A new approach to preventing relapse. New York: Guilford Press.

Shanafelt, T. D., Sloan, J. A., \& Habermann, T. M. (2003). The wellbeing of physicians. American Journal of Medicine, 114, 513-519. https://doi.org/10.1016/s0002-9343(03)00117-7.

Shanafelt, T. D., Balch, C. M., Bechamps, G. J., Russell, T., Dyrbye, L., Satele, D., et al. (2009). Burnout and career satisfaction among American surgeons. Annals of Surgery, 250, 463-471. https://doi. org/10.1097/sla.0b013e3181ac4dfd.

Shapiro, S. L., Astin, J. A., Bishop, S. R., \& Cordova, M. (2005). Mindfulness-based stress reduction for health care professionals: 
Results from a randomized controlled trial. International Journal of Stress Management, 12, 164-176. https://doi.org/10.1037/10725245.12.2.164.

Shapiro, S. L., Carlson, L. E., Astin, J. A., \& Freedman, B. (2006). Mechanisms of mindfulness. Journal of Clinical Psychology, 62(3), 373-286. https://doi.org/10.1002/jclp.20237.

Shapiro, S. L., Brown, K., \& Biegel, G. (2007). Self-care for health professionals: Effects of MBSR on the mental of therapists in training. Training and Education in Professional Psychology, 1(2), 105115. https://doi.org/10.1037/1931-3918.1.2.105.

Sharma, M., \& Rush, S. E. (2014). Mindfulness-based stress reduction as a stress management intervention for healthy individuals: A systematic review. Journal of Evidenced-Based Complementary and Alternative Medicine, 19(4), 271-286. https://doi.org/10.1177/ 2156587214543143.

Smith, S. A. (2014). Mindfulness-based stress reduction: An intervention to enhance effectiveness of nurses' coping with work-related stress. International Journal of Nursing Knowledge, 25(2), 119-130. https://doi.org/10.1111/2047-3095.12025.

Spickard, A., Gabbe, S. G., \& Christensen, J. F. (2002). Mid-career burnout in generalist and specialist physicians. Journal of the American Medical Association, 288, 1447-1450. https://doi.org/10.1001/ jama.288.12.1447.

Spinelli, C., Wisener, M., \& Khoury, B. (2019). Mindfulness training for healthcare professionals and trainees: A meta-analysis of randomized controlled trials. Journal of Psychosomatic Research, 120, 29 38. https://doi.org/10.1016/j.jpsychores.2019.03.003.

*Suyi, Y., Meredith, P., \& Khan, A. (2017). Effectiveness of mindfulness intervention in reducing stress and burnout for mental health professionals in Singapore. Explore, 13(5), 319-326. https://doi.org/10. 1016/j.explore.2017.06.001.

Thomas, B. H., Ciliska, D., Dobbins, M., \& Micucci, S. (2004). A process for systematically reviewing the literature: Providing the research evidence for public health nursing interventions. Worldviews on Evidenced Based Nursing, 1(3), 176-184. https://doi.org/10.1111/ j.1524-475x.2004.04006.x.

*Trowbridge, K., Lawson, L.M., Andrews, S., Pecora, J., \& Boyd, S. (2017). Preliminary investigation of workplace-provided compressed mindfulness-based stress reduction with paediatric social workers. Health and Social Work, 42(4), 207-2014. https://doi. org/10.1093/hsw/hlx038.

Vahey, D. C., Aiken, L. H., Sloane, D. M., Clarke, S. P., \& Vargas, D. (2004). Nurse burnout and patient satisfaction. Medical Care, 42, 57-66. https://doi.org/10.1097/01.mlr.0000109126.50398.5a.

*Van Wietmarschen, H., Tjaden, B., van Vilet, M., Battjes-Fries, M., \& Jong, M. (2018). Effects of mindfulness training on perceived stress, self-compassion and self-reflection of primary care physicians: A mixed-methods study. BJGP Open, 2(4): 1-11. https://doi.org/10. 3399/bjgpopen $18 \times 101621$.

*Verweij, H., Waumans, R.C., Smeijers, D., Lucassen, P.L.B.J., Donders, A.R.T., van der Horst, H.E., \& Speckens, A.E.M. (2016). Mindfulness-based stress reduction in GPs: Results of a controlled mixed methods pilot study in Dutch primary care. British Journal of General Practice Research, 66, 99-105. https:// doi.org/10.3399/bjgp16x683497.

Virgili, M. (2013). Mindfulness-based interventions reduce psychological distress in working adults: A meta-analysis of intervention studies. Mindfulness, 6, 326-337. https://doi.org/10.1007/s12671-0130264-0.

*Wang, S.C., Wang, L.Y., Shih, S.M., Chang, S.C., \& Fan, S.Y. (2017). The effects of mindfulness-based stress reduction on hospital nursing staff. Applied Nursing Research, 38, 124-128. https://doi.org/ 10.1016/j.apnr.2017.09.014.

Weinberg, A., \& Creed, F. (2000). Stress and psychiatric disorder in healthcare professionals and hospital staff. Lancet, 355, 533-537. https://doi.org/10.1016/s0140-6736(99)07366-3.

Williams, E. S., Manwell, L. B., Konrad, T. R., \& Linzwer, M. (2007). The relationship of organisational culture, stress, satisfaction, and burnout and physician-reported error and suboptimal patient care: Results from the MEMO study. Health Care Management Review, 32, 203-212. https://doi.org/10.1097/01.hmr.0000281626.28363.59.

Publisher's Note Springer Nature remains neutral with regard to jurisdictional claims in published maps and institutional affiliations. 